\title{
Observation of $\Lambda_{\mathrm{b}}^{0} \rightarrow \psi(2 \mathrm{~S}) \mathrm{pK}^{-}$and
} $\Lambda_{\mathrm{b}}^{0} \rightarrow \mathrm{J} / \psi \pi^{+} \pi^{-} \mathrm{pK}^{-}$decays and a measurement of the $\Lambda_{\mathrm{b}}^{0}$ baryon mass

\section{LHCb}

\section{The LHCb collaboration}

E-mail: Ivan.Belyaev@cern.ch

AbStRaCt: The decays $\Lambda_{\mathrm{b}}^{0} \rightarrow \psi(2 \mathrm{~S}) \mathrm{pK}^{-}$and $\Lambda_{\mathrm{b}}^{0} \rightarrow \mathrm{J} / \psi \pi^{+} \pi^{-} \mathrm{pK}^{-}$are observed in a data sample corresponding to an integrated luminosity of $3 \mathrm{fb}^{-1}$, collected in proton-proton collisions at 7 and $8 \mathrm{TeV}$ centre-of-mass energies by the LHCb detector. The $\psi(2 \mathrm{~S})$ mesons are reconstructed through the decay modes $\psi(2 \mathrm{~S}) \rightarrow \mu^{+} \mu^{-}$and $\psi(2 \mathrm{~S}) \rightarrow \mathrm{J} / \psi \pi^{+} \pi^{-}$. The branching fractions relative to that of $\Lambda_{\mathrm{b}}^{0} \rightarrow \mathrm{J} / \psi \mathrm{pK}^{-}$are measured to be

$$
\begin{aligned}
\frac{\mathcal{B}\left(\Lambda_{\mathrm{b}}^{0} \rightarrow \psi(2 \mathrm{~S}) \mathrm{pK}^{-}\right)}{\mathcal{B}\left(\Lambda_{\mathrm{b}}^{0} \rightarrow \mathrm{J} / \psi \mathrm{pK}^{-}\right)} & =(20.70 \pm 0.76 \pm 0.46 \pm 0.37) \times 10^{-2}, \\
\frac{\mathcal{B}\left(\Lambda_{\mathrm{b}}^{0} \rightarrow \mathrm{J} / \psi \pi^{+} \pi^{-} \mathrm{pK}^{-}\right)}{\mathcal{B}\left(\Lambda_{\mathrm{b}}^{0} \rightarrow \mathrm{J} / \psi \mathrm{pK}^{-}\right)} & =(20.86 \pm 0.96 \pm 1.34) \times 10^{-2},
\end{aligned}
$$

where the first uncertainties are statistical, the second are systematic and the third is related to the knowledge of $\mathrm{J} / \psi$ and $\psi(2 \mathrm{~S})$ branching fractions. The mass of the $\Lambda_{\mathrm{b}}^{0}$ baryon is measured to be

$$
M\left(\Lambda_{\mathrm{b}}^{0}\right)=5619.65 \pm 0.17 \pm 0.17 \mathrm{MeV} / c^{2},
$$

where the uncertainties are statistical and systematic.

KeYwords: B physics, Flavor physics, Hadron-Hadron scattering (experiments), Particle and resonance production, Spectroscopy

ARXiv EPrint: 1603.06961 


\section{Contents}

1 Introduction 1

2 Detector and simulation $\quad 2$

3 Event selection 3

4 Measurement of branching fractions 4

4.1 Signal yields and efficiencies 4

$\begin{array}{lll}4.2 & \text { Systematic uncertainties } & 6\end{array}$

4.3 Results 8

5 Measurement of $\Lambda_{\mathrm{b}}^{0}$ baryon mass $\quad 9$

6 Results and summary $\quad 12$

$\begin{array}{ll}\text { The LHCb collaboration } & 17\end{array}$

\section{Introduction}

The $\Lambda_{\mathrm{b}}^{0}$ baryon is the isospin singlet ground state of a bottom quark and two light quarks. The rich phenomenology associated with decays of bottom baryons allows many measurements of masses, lifetimes and branching fractions, which test the theoretical understanding of weak decays of heavy hadrons in the framework of heavy quark effective theory (HQET) and the underlying QCD physics [1,2]. At the Tevatron, properties of the $\Lambda_{\mathrm{b}}^{0}$ baryon, such as mass and lifetime, have been measured using two-body modes, specifically $\Lambda_{\mathrm{b}}^{0} \rightarrow \mathrm{J} / \psi \Lambda^{0}$ and $\Lambda_{\mathrm{b}}^{0} \rightarrow \Lambda_{\mathrm{c}}^{+} \pi^{-}$decays [3-5]. ${ }^{1}$ The high production rate of b quarks at the Large Hadron Collider (LHC), along with the excellent momentum and mass resolution and the hadron identification capabilities of the LHCb detector, open up a host of multibody and Cabibbo-suppressed decay channels of $\Lambda_{\mathrm{b}}^{0}$ baryons, e.g. the decays $\Lambda_{\mathrm{b}}^{0} \rightarrow \mathrm{D}^{0} \mathrm{pK}^{-}, \Lambda_{\mathrm{b}}^{0} \rightarrow \Lambda_{\mathrm{c}}^{+} \mathrm{K}^{-}[6], \Lambda_{\mathrm{b}}^{0} \rightarrow \Lambda_{\mathrm{c}}^{+} \mathrm{D}^{-}, \Lambda_{\mathrm{b}}^{0} \rightarrow \Lambda_{\mathrm{c}}^{+} \mathrm{D}_{\mathrm{s}}^{-}$[7] and $\Lambda_{\mathrm{b}}^{0} \rightarrow \mathrm{J} / \psi \mathrm{p} \pi^{-}$[8]. The high signal yield of the $\Lambda_{\mathrm{b}}^{0} \rightarrow \mathrm{J} / \psi \mathrm{pK}^{-}$decay [9] allowed the precise measurement of the $\Lambda_{\mathrm{b}}^{0}$ lifetime $[10,11]$. The recent analysis of this decay mode uncovered a double resonant structure in the $\mathrm{J} / \psi \mathrm{p}$ system consistent with two pentaquark states [12]. LHCb has also measured several B meson decays into final states with charmonia [13-18]. The first observation of $\Lambda_{\mathrm{b}}^{0}$ decays to excited charmonium, the $\Lambda_{\mathrm{b}}^{0} \rightarrow \psi(2 \mathrm{~S}) \Lambda^{0}$ decay, has been presented by the ATLAS collaboration [19]. An experimental investigation of other similar multibody decays of the $\Lambda_{\mathrm{b}}^{0}$ baryon should lead to deeper insights into QCD.

\footnotetext{
${ }^{1}$ The inclusion of charge-conjugate modes is implied throughout this paper.
} 
In this paper, the first observations of the decays $\Lambda_{\mathrm{b}}^{0} \rightarrow \psi(2 \mathrm{~S}) \mathrm{pK}^{-}$and $\Lambda_{\mathrm{b}}^{0} \rightarrow \mathrm{J} / \psi \pi^{+} \pi^{-} \mathrm{pK}^{-}$are reported, where $\psi(2 \mathrm{~S})$ mesons are reconstructed in the final states $\mu^{+} \mu^{-}$and $\mathrm{J} / \psi \pi^{+} \pi^{-}$. The ratios of the branching fractions of these decays to that of the normalization decay $\Lambda_{\mathrm{b}}^{0} \rightarrow \mathrm{J} / \psi \mathrm{pK}^{-}$,

$$
\begin{aligned}
R^{\psi(2 \mathrm{~S})} & \equiv \frac{\mathcal{B}\left(\Lambda_{\mathrm{b}}^{0} \rightarrow \psi(2 \mathrm{~S}) \mathrm{pK}^{-}\right)}{\mathcal{B}\left(\Lambda_{\mathrm{b}}^{0} \rightarrow \mathrm{J} / \psi \mathrm{pK}^{-}\right)}, \\
R^{\mathrm{J} / \psi \pi^{+} \pi^{-}} & \equiv \frac{\mathcal{B}\left(\Lambda_{\mathrm{b}}^{0} \rightarrow \mathrm{J} / \psi \pi^{+} \pi^{-} \mathrm{pK}^{-}\right)}{\mathcal{B}\left(\Lambda_{\mathrm{b}}^{0} \rightarrow \mathrm{J} / \psi \mathrm{pK}^{-}\right)},
\end{aligned}
$$

are measured. In measuring the branching fraction of $\Lambda_{\mathrm{b}}^{0} \rightarrow \mathrm{J} / \psi \pi^{+} \pi^{-} \mathrm{pK}^{-}$decays, contributions via intermediate resonances, such as $\Lambda_{\mathrm{b}}^{0} \rightarrow \psi(2 \mathrm{~S}) \mathrm{pK}^{-}$, are implicitly included. The low energy release in these decays allows a precise determination of the $\Lambda_{\mathrm{b}}^{0}$ mass with a small systematic uncertainty.

This study is based on a data sample corresponding to an integrated luminosity of $3 \mathrm{fb}^{-1}$, collected with the LHCb detector in pp collisions at centre-of-mass energies $\sqrt{s}=7$ and $8 \mathrm{TeV}$.

\section{Detector and simulation}

The LHCb detector $[20,21]$ is a single-arm forward spectrometer covering the pseudorapidity range $2<\eta<5$, designed for the study of particles containing b or c quarks. The detector includes a high-precision tracking system consisting of a silicon-strip vertex detector surrounding the pp interaction region, a large-area silicon-strip detector located upstream of a dipole magnet with a bending power of about $4 \mathrm{Tm}$, and three stations of silicon-strip detectors and straw drift tubes placed downstream of the magnet. The polarity of the dipole magnet is reversed periodically throughout data-taking. The tracking system provides a measurement of the momentum, $p$, of charged particles with a relative uncertainty that varies from $0.5 \%$ at low momentum to $1.0 \%$ at $200 \mathrm{GeV} / c$. The minimum distance of a track to a primary vertex $(\mathrm{PV})$, the impact parameter, is measured with a resolution of $\left(15+29 / p_{\mathrm{T}}\right) \mu \mathrm{m}$, where $p_{\mathrm{T}}$ is the component of the momentum transverse to the beam, in $\mathrm{GeV} / c$ [22]. Large samples of $\mathrm{B}^{+} \rightarrow \mathrm{J} / \psi \mathrm{K}^{+}$and $\mathrm{J} / \psi \rightarrow \mu^{+} \mu^{-}$decays, collected concurrently with the data set, were used to calibrate the momentum scale of the spectrometer to a precision of $0.03 \%$ [23].

Different types of charged hadrons are distinguished using information from two ringimaging Cherenkov detectors (RICH). Photons, electrons and hadrons are identified by a calorimeter system consisting of scintillating-pad and preshower detectors, an electromagnetic calorimeter and a hadronic calorimeter. Muons are identified by a system composed of alternating layers of iron and multiwire proportional chambers.

The trigger [24] comprises two stages. Events are first required to pass the hardware trigger, which selects muon candidates with $p_{\mathrm{T}}>1.48(1.76) \mathrm{GeV} / c$ or pairs of opposite-sign muon candidates with a requirement that the product of the muon transverse momenta is larger than $1.7(2.6) \mathrm{GeV}^{2} / c^{2}$ for data collected at $\sqrt{s}=7(8) \mathrm{TeV}$. The subsequent software trigger is composed of two stages, the first of which performs a partial event reconstruction, 
while full event reconstruction is done at the second stage. At the first stage of the software trigger the invariant mass of well-reconstructed pairs of oppositely charged muons forming a good-quality two-track vertex is required to exceed $2.7 \mathrm{GeV} / c^{2}$, and the two-track vertex is required to be significantly displaced from all PVs.

The analysis technique reported below has been validated using simulated events. The pp collisions are generated using PYTHIA $[25,26]$ with a specific LHCb configuration [27]. Decays of hadronic particles are described by EvTGen [28], in which final-state radiation is generated using Pнотоs [29]. The interaction of the generated particles with the detector, and its response, are implemented using the GEANT4 toolkit [30, 31] as described in ref. [32].

\section{Event selection}

The decays $\Lambda_{\mathrm{b}}^{0} \rightarrow \psi(2 \mathrm{~S}) \mathrm{pK}^{-}, \Lambda_{\mathrm{b}}^{0} \rightarrow \mathrm{J} / \psi \pi^{+} \pi^{-} \mathrm{pK}^{-}$and $\Lambda_{\mathrm{b}}^{0} \rightarrow \mathrm{J} / \psi \mathrm{pK}^{-}$are reconstructed using decay modes $\psi(2 \mathrm{~S}) \rightarrow \mu^{+} \mu^{-}, \psi(2 \mathrm{~S}) \rightarrow \mathrm{J} / \psi \pi^{+} \pi^{-}$and $\mathrm{J} / \psi \rightarrow \mu^{+} \mu^{-}$. Common selection criteria, based on those used in refs. [17, 33], are used for all channels, except for those related to the selection of two additional pions in the $\Lambda_{\mathrm{b}}^{0} \rightarrow \mathrm{J} / \psi \pi^{+} \pi^{-} \mathrm{pK} \mathrm{K}^{-}$and $\Lambda_{\mathrm{b}}^{0} \rightarrow \psi(2 \mathrm{~S})\left[\rightarrow \mathrm{J} / \psi \pi^{+} \pi^{-}\right] \mathrm{pK}^{-}$channels.

Muon, proton, kaon and pion candidates are selected from well-reconstructed tracks within the acceptance of the spectrometer that are identified using information from the RICH, calorimeter and muon detectors [34, 35]. Muons, protons, kaons and pions are required to have a transverse momentum larger than 550, 800, 500 and $200 \mathrm{MeV} / c$, respectively. To allow good particle identification, kaons and pions are required to have a momentum between $3.2 \mathrm{GeV} / c$ and $150 \mathrm{GeV} / c$ whilst protons must have a momentum between $10 \mathrm{GeV} / c$ and $150 \mathrm{GeV} / c$. To reduce combinatorial background involving tracks from the primary pp interaction vertices, only tracks that exceed a minimum impact parameter $\chi^{2}$ with respect to every PV are used. The impact parameter $\chi^{2}$ is defined as the difference between the $\chi^{2}$ of the PV reconstructed with and without the considered particle.

Pairs of oppositely-charged muons originating from a common vertex are combined to form $\mathrm{J} / \psi \rightarrow \mu^{+} \mu^{-}$or $\psi(2 \mathrm{~S}) \rightarrow \mu^{+} \mu^{-}$candidates. The resulting dimuon candidates are required to have an invariant mass between $-5 \sigma$ and $+3 \sigma$ around the known $\mathrm{J} / \psi$ or $\psi(2 \mathrm{~S})$ masses [36], where $\sigma$ is the mass resolution. An asymmetric mass interval is chosen to include part of the low-mass tail due to final-state radiation.

Candidate $\Lambda_{\mathrm{b}}^{0}$ baryons are formed from $\mathrm{J} / \psi \mathrm{pK}^{-}, \psi(2 \mathrm{~S}) \mathrm{pK}^{-}$and $\mathrm{J} / \psi \pi^{+} \pi^{-} \mathrm{pK}^{-}$combinations. Each candidate is associated with the PV with respect to which it has the smallest impact parameter significance. The $\Lambda_{\mathrm{b}}^{0}$ mass resolution is improved by employing a kinematic fit [37] that constrains the mass of the $\mathrm{J} / \psi$ candidate to its known value and requires the momentum of the $\Lambda_{\mathrm{b}}^{0}$ candidate to point back to the PV. A requirement on the quality of this fit is applied to further suppress combinatorial background. Finally, the measured decay time of the $\Lambda_{\mathrm{b}}^{0}$ candidate, calculated with respect to the associated primary vertex, is required to be between 0.5 and $6.7 \mathrm{ps}$. The lower limit is used to suppress background from particles coming from the PV while the upper limit removes poorly reconstructed candidates. 

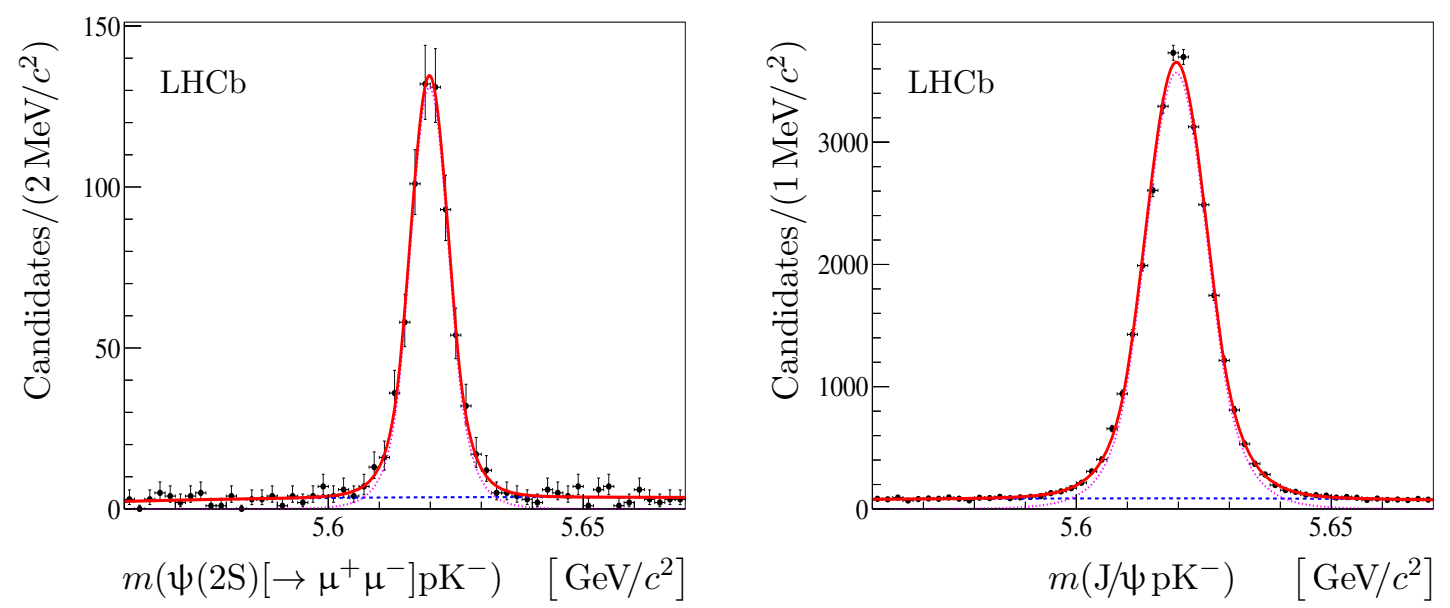

Figure 1. Mass distributions of selected (left) $\Lambda_{\mathrm{b}}^{0} \rightarrow \psi(2 \mathrm{~S})\left[\rightarrow \mu^{+} \mu^{-}\right] \mathrm{pK}^{-}$and (right) $\Lambda_{\mathrm{b}}^{0} \rightarrow \mathrm{J} / \psi \mathrm{pK}{ }^{-}$ candidates. The total fit function (solid red), the $\Lambda_{\mathrm{b}}^{0}$ signal contribution (dotted magenta) and the combinatorial background (dashed blue) are shown. The error bars show $68 \%$ Poissonian confidence intervals.

To suppress cross-feed from decays of the $\mathrm{B}_{\mathrm{s}}^{0}$ meson into $\mathrm{J} / \psi \mathrm{K}^{-} \mathrm{K}^{+}, \psi(2 \mathrm{~S}) \mathrm{K}^{-} \mathrm{K}^{+}$and $\mathrm{J} / \psi \pi^{+} \pi^{-} \mathrm{K}^{-} \mathrm{K}^{+}$final states, with the positively-charged kaon misidentified as a proton, a veto on the $\Lambda_{\mathrm{b}}^{0}$ candidate mass, recalculated with a kaon mass hypothesis for the proton, is applied. Any candidate with a recalculated mass consistent with the nominal $\mathrm{B}_{\mathrm{s}}^{0}$ mass is rejected. A similar veto is applied to suppress cross-feed from decays of $\mathrm{B}^{0}$ mesons into $\mathrm{J} / \psi \mathrm{K}^{-} \pi^{+}, \psi(2 \mathrm{~S}) \mathrm{K}^{-} \pi^{+}$and $\mathrm{J} / \psi \pi^{-} \pi^{+} \pi^{+} \mathrm{K}^{-}$decays with the positively-charged pion misidentified as a proton.

\section{Measurement of branching fractions}

\subsection{Signal yields and efficiencies}

The mass distributions for selected $\Lambda_{\mathrm{b}}^{0} \rightarrow \psi(2 \mathrm{~S})\left[\rightarrow \mu^{+} \mu^{-}\right] \mathrm{pK}^{-}$candidates and candidates for the normalization channel $\Lambda_{\mathrm{b}}^{0} \rightarrow \mathrm{J} / \psi \mathrm{pK}^{-}$are shown in figure 1. Signal yields are determined using unbinned extended maximum likelihood fits to these distributions. The signal is modelled with a modified Gaussian function with power-law tails on both sides [38, 39], where the tail parameters are fixed from simulation and the mass resolution parameter is allowed to vary. The background is modelled with an exponential function multiplied by a first-order polynomial. The resolution parameters obtained from the fits are found to be $3.82 \pm 0.17 \mathrm{MeV} / c^{2}$ for the channel $\Lambda_{\mathrm{b}}^{0} \rightarrow \psi(2 \mathrm{~S})\left[\rightarrow \mu^{+} \mu^{-}\right] \mathrm{pK}^{-}$and $6.12 \pm 0.05 \mathrm{MeV} / c^{2}$ for $\Lambda_{\mathrm{b}}^{0} \rightarrow \mathrm{J} / \psi \mathrm{pK}^{-}$, in good agreement with expectations from simulation.

The mass distribution for selected $\Lambda_{\mathrm{b}}^{0} \rightarrow \mathrm{J} / \psi \pi^{+} \pi^{-} \mathrm{pK}^{-}$candidates is shown in figure 2(left), along with the result of an unbinned extended maximum likelihood fit using the model described above. The mass resolution parameter obtained from the fit is $4.72 \pm 0.23 \mathrm{MeV} / c^{2}$. The mass distribution of the $\mathrm{J} / \psi \pi^{+} \pi^{-}$system from signal $\Lambda_{\mathrm{b}}^{0} \rightarrow \mathrm{J} / \psi \pi^{+} \pi^{-} \mathrm{pK}^{-}$decays is presented in figure 2(right) in the region $3.67<m\left(\mathrm{~J} / \psi \pi^{+} \pi^{-}\right)<3.7 \mathrm{GeV} / c^{2}$. 

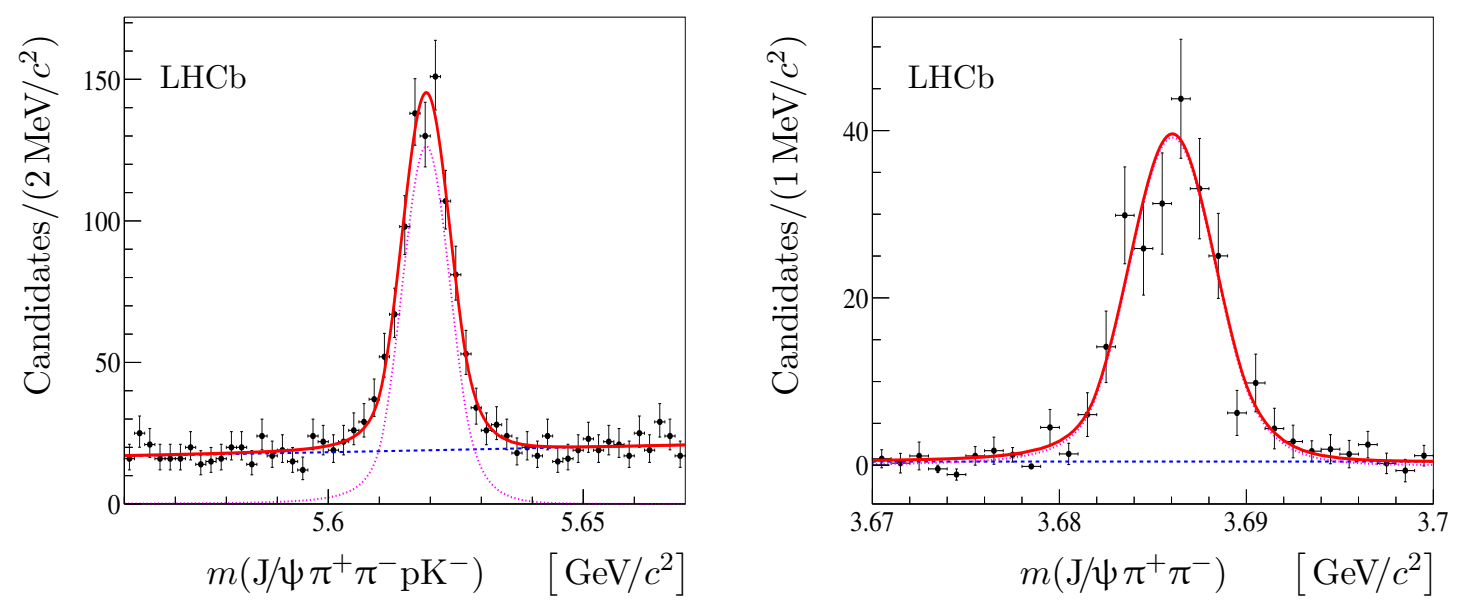

Figure 2. Left: mass distribution of selected $\Lambda_{\mathrm{b}}^{0} \rightarrow \mathrm{J} / \psi \pi^{+} \pi^{-} \mathrm{pK} \mathrm{K}^{-}$candidates. Right: backgroundsubtracted $\mathrm{J} / \psi \pi^{+} \pi^{-}$mass distribution for that mode. The total fit function and the signal contributions are shown by solid red and dotted magenta lines, respectively. The combinatorial background in the left plot and nonresonant contribution in the right plot are shown by dashed blue lines.

\begin{tabular}{|lc|}
\hline Channel & $N\left(\Lambda_{\mathrm{b}}^{0}\right)$ \\
\hline$\Lambda_{\mathrm{b}}^{0} \rightarrow \mathrm{J} / \psi \mathrm{pK}^{-}$ & $28834 \pm 204$ \\
$\Lambda_{\mathrm{b}}^{0} \rightarrow \psi(2 \mathrm{~S})\left[\rightarrow \mu^{+} \mu^{-}\right] \mathrm{pK}^{-}$ & $665 \pm 28$ \\
$\Lambda_{\mathrm{b}}^{0} \rightarrow \psi(2 \mathrm{~S})\left[\rightarrow \mathrm{J} / \psi \pi^{+} \pi^{-}\right] \mathrm{pK}^{-}$ & $231 \pm 17$ \\
$\Lambda_{\mathrm{b}}^{0} \rightarrow \mathrm{J} / \psi \pi^{+} \pi^{-} \mathrm{pK}^{-}$ & $793 \pm 36$ \\
\hline
\end{tabular}

Table 1. Signal yields of $\Lambda_{\mathrm{b}}^{0}$ decay channels. Uncertainties are statistical only.

The background subtraction is performed with the sPlot technique [40] using the $\mathrm{J} / \psi \pi^{+} \pi^{-} \mathrm{pK}^{-}$mass as the discriminating variable. The signal yield of $\Lambda_{\mathrm{b}}^{0} \rightarrow \psi(2 \mathrm{~S})\left[\rightarrow \mathrm{J} / \psi \pi^{+} \pi^{-}\right] \mathrm{pK}^{-}$decays is determined using an unbinned extended maximum likelihood fit to the $\mathrm{J} / \psi \pi^{+} \pi^{-}$invariant mass distribution. The $\psi(2 \mathrm{~S})$ component is modelled with a modified Gaussian function with power-law tails on both sides, where the tail parameters are fixed from simulation. The nonresonant component is taken to be constant. The mass resolution parameter obtained from the fit is $2.29 \pm 0.17 \mathrm{MeV} / c^{2}$. The signal yields are summarized in table 1.

The ratio of branching fractions $R^{\psi(2 \mathrm{~S})}$, defined in eq. (1.1), is measured in two different decay modes,

$$
\begin{aligned}
\left.R^{\psi(2 \mathrm{~S})}\right|_{\psi(2 \mathrm{~S}) \rightarrow \mu^{+} \mu^{-}} & =\frac{N_{\psi(2 \mathrm{~S}) \rightarrow \mu^{+} \mu^{-}}}{N_{\mathrm{J} / \psi}} \times \frac{\varepsilon_{\mathrm{J} / \psi}^{\Lambda_{\mathrm{b}}^{0}}}{\varepsilon_{\psi(2 \mathrm{~b}) \rightarrow \mu^{+} \mu^{-}}^{\Lambda_{\mathrm{b}}^{0}}} \times \frac{\mathcal{B}\left(\mathrm{J} / \psi \rightarrow \mu^{+} \mu^{-}\right)}{\mathcal{B}\left(\psi(2 \mathrm{~S}) \rightarrow \mu^{+} \mu^{-}\right)}, \\
\left.R^{\psi(2 \mathrm{~S})}\right|_{\psi(2 \mathrm{~S}) \rightarrow \mathrm{J} / \psi \pi^{+} \pi^{-}} & =\frac{N_{\psi(2 \mathrm{~S}) \rightarrow \mathrm{J} / \psi \pi^{+} \pi^{-}}}{N_{\mathrm{J} / \psi}} \times \frac{\varepsilon_{\mathrm{J} / \psi}^{\Lambda_{\mathrm{b}}^{0}}}{\varepsilon_{\psi(2 \mathrm{~S}) \rightarrow \mathrm{J} / \psi \pi^{+} \pi^{-}}^{\Lambda_{\mathrm{b}}^{0}}} \times \frac{1}{\mathcal{B}\left(\psi(2 \mathrm{~S}) \rightarrow \mathrm{J} / \psi \pi^{+} \pi^{-}\right)},
\end{aligned}
$$




\begin{tabular}{|cc|}
\hline & Value \\
\hline$\varepsilon_{\mathrm{J} / \psi}^{\Lambda_{\mathrm{b}}^{0}} / \varepsilon_{\psi(2 \mathrm{~S}) \rightarrow \mu^{+} \mu^{-}}^{\Lambda_{\mathrm{b}}^{0}}$ & $1.188 \pm 0.006$ \\
$\varepsilon_{\mathrm{J} / \psi}^{\Lambda_{\mathrm{b}}^{0}} / \varepsilon_{\psi(2 \mathrm{~S}) \rightarrow \mathrm{J} / \psi \pi^{+} \pi^{-}}^{\Lambda_{\mathrm{b}}^{0}}$ & $8.84 \pm 0.05$ \\
$\varepsilon_{\mathrm{J} / \psi}^{\Lambda_{\mathrm{b}}^{0}} / \varepsilon_{\mathrm{J} / \psi \pi^{+} \pi^{-}}^{\Lambda_{\mathrm{b}}^{0}}$ & $7.59 \pm 0.04$ \\
\hline
\end{tabular}

Table 2. Ratios of efficiencies. The uncertainties reflect the limited size of the simulation sample.

and the ratio $R^{\mathrm{J} / \psi \pi^{+} \pi^{-}}$, defined in eq. (1.2), is measured as

$$
R^{\mathrm{J} / \psi \pi^{+} \pi^{-}}=\frac{N_{\mathrm{J} / \psi \pi^{+} \pi^{-}}}{N_{\mathrm{J} / \psi}} \times \frac{\varepsilon_{\mathrm{J} / \psi}^{\Lambda_{\mathrm{b}}^{0}}}{\varepsilon_{\mathrm{J} / \psi \pi^{+} \pi^{-}}^{\Lambda_{\mathrm{b}}^{0}}},
$$

where $N_{\mathrm{X}}$ represents the observed signal yield and $\varepsilon_{\mathrm{X}}^{\Lambda_{\mathrm{b}}^{0}}$ denotes the efficiency for the decay $\Lambda_{\mathrm{b}}^{0} \rightarrow \mathrm{XpK}^{-}$. The ratio $\frac{\mathcal{B}\left(\mathrm{J} / \psi \rightarrow \mu^{+} \mu^{-}\right)}{\mathcal{B}\left(\psi(2 \mathrm{~S}) \rightarrow \mu^{+} \mu^{-}\right)}$is taken to be equal to the more precisely measured ratio of dielectron branching fractions, $\frac{\mathcal{B}\left(\mathrm{J} / \psi \rightarrow \mathrm{e}^{+} \mathrm{e}^{-}\right)}{\mathcal{B}\left(\psi(2 \mathrm{~S}) \rightarrow \mathrm{e}^{+} \mathrm{e}^{-}\right)}=7.57 \pm 0.17$ [36]. For the $\psi(2 \mathrm{~S}) \rightarrow \mathrm{J} / \psi \pi^{+} \pi^{-}$branching fraction the world average $(34.46 \pm 0.30) \%$ [36] is taken.

The efficiency is defined as the product of the geometric acceptance and the detection, reconstruction, selection and trigger efficiencies. The efficiencies for hadron identification as functions of kinematic parameters and event multiplicity are determined from data using calibration samples of low-background decays: $\mathrm{D}^{*+} \rightarrow \mathrm{D}^{0} \pi^{+}$followed by $\mathrm{D}^{0} \rightarrow \mathrm{K}^{-} \pi^{-}$ for kaons and pions, and $\Lambda^{0} \rightarrow \mathrm{p} \pi^{-}$and $\Lambda_{\mathrm{c}}^{+} \rightarrow \mathrm{pK}{ }^{-} \pi^{+}$for protons [34]. The remaining efficiencies are determined using simulation.

In the simulation of $\Lambda_{\mathrm{b}}^{0} \rightarrow \mathrm{J} / \psi \mathrm{pK}^{-}$decays, the model established in ref. [12] that includes pentaquark contributions is used, while in the simulation of the other decay modes the events are generated uniformly in phase space. The simulation is corrected to reproduce the transverse momentum and rapidity distributions of the $\Lambda_{\mathrm{b}}^{0}$ baryons observed in data [9] and to account for small discrepancies between data and simulation in the reconstruction of charged tracks [41]. The ratios of efficiencies to those in the $\Lambda_{\mathrm{b}}^{0} \rightarrow \mathrm{J} / \psi \mathrm{pK}^{-}$channel are presented in table 2 .

\subsection{Systematic uncertainties}

Most systematic uncertainties cancel in the measurements of the ratios of branching fractions, notably those related to the reconstruction, identification and trigger efficiencies of the $\mathrm{J} / \psi \rightarrow \mu^{+} \mu^{-}$and $\psi(2 \mathrm{~S}) \rightarrow \mu^{+} \mu^{-}$candidates [13], due to the similarity of the muon and dimuon spectra for these modes. The remaining systematic uncertainties are summarized in table 3 and discussed below.

Alternative parametrizations for the signal and background are used to estimate the systematic uncertainties related to the fit model. A modified Novosibirsk function [42], 


\begin{tabular}{|lccc|}
\hline Source & $\left.R^{\psi(2 \mathrm{~S})}\right|_{\psi(2 \mathrm{~S}) \rightarrow \mu^{+} \mu^{-}}$ & $\left.R^{\psi(2 \mathrm{~S})}\right|_{\psi(2 \mathrm{~S}) \rightarrow \mathrm{J} / \psi \pi^{+} \pi^{-}}$ & $R^{\mathrm{J} / \psi \pi^{+} \pi^{-}}$ \\
\hline Fit model & 0.8 & 3.0 & 3.5 \\
Cross-feed & 0.8 & 0.9 & 0.9 \\
Efficiency calculation: & & & \\
$\Lambda_{\mathrm{b}}^{0}$ decay model & 0.3 & 0.8 & 0.8 \\
Reconstruction of additional pions: & & & \\
$\quad$ Hadron interaction & - & $2 \times 2.0$ & $2 \times 2.0$ \\
$\quad$ Track efficiency correction & - & 3.2 & 2.7 \\
Hadron identification & 0.1 & 0.1 & 0.2 \\
Trigger & 1.1 & 1.1 & 1.1 \\
Selection criteria & 0.6 & 0.9 & 0.2 \\
Simulation sample size & 1.0 & 1.6 & 1.7 \\
\hline Sum in quadrature & 2.0 & 6.4 & 6.4 \\
\hline
\end{tabular}

Table 3. Systematic uncertainties (in \%) on the ratios of branching fractions $R^{\psi(2 \mathrm{~S})}$ and $R^{\mathrm{J} / \psi \pi^{+} \pi^{-}}$.

an Apolonios function [43], an asymmetric variant of the Apolonios function and the Student's t-distribution are used for the $\Lambda_{\mathrm{b}}^{0}$ signal shape, and an exponential function multiplied by a second-order polynomial is used for the background. The ratio of event yields is remeasured with the cross-check models, and the maximum deviation with respect to the nominal value is assigned as a systematic uncertainty.

The uncertainty associated with the $\mathrm{B}_{\mathrm{s}}^{0}$ and $\mathrm{B}^{0}$ cross-feed is estimated by varying the widths of the rejected regions and recomputing the signal yields, taking into account the changes in efficiencies. As an additional cross-check, a veto is applied also on possible contributions from $\bar{\Lambda}_{\mathrm{b}}^{0} \rightarrow \mathrm{J} / \psi \overline{\mathrm{p}} \mathrm{K}^{+}, \bar{\Lambda}_{\mathrm{b}}^{0} \rightarrow \psi(2 \mathrm{~S}) \overline{\mathrm{p}} \mathrm{K}^{+}$and $\bar{\Lambda}_{\mathrm{b}}^{0} \rightarrow \mathrm{J} / \psi \pi^{+} \pi^{-} \overline{\mathrm{p}} \mathrm{K}^{+}$decays where the positive kaon is misidentified as a proton and the antiproton is misidentified as a negative kaon. The maximum of the observed differences from the nominal values is assigned as the corresponding systematic uncertainty.

The remaining systematic uncertainties are associated with the efficiency determination. The systematic uncertainty related to the decay model for $\Lambda_{\mathrm{b}}^{0} \rightarrow \psi(2 \mathrm{~S}) \mathrm{pK}^{-}$and $\Lambda_{\mathrm{b}}^{0} \rightarrow \mathrm{J} / \psi \pi^{+} \pi^{-} \mathrm{pK}^{-}$decays is estimated using the simulated samples, corrected to reproduce the invariant mass of the $\mathrm{pK}^{-}$and $\psi(2 \mathrm{~S}) \mathrm{p}$ or $\mathrm{J} / \psi \pi^{+} \pi^{-}$p systems observed in data. The largest change in efficiency is taken as the corresponding systematic uncertainty.

The decay modes $\Lambda_{\mathrm{b}}^{0} \rightarrow \mathrm{J} / \psi \pi^{+} \pi^{-} \mathrm{pK}^{-}$and $\Lambda_{\mathrm{b}}^{0} \rightarrow \psi(2 \mathrm{~S})\left[\rightarrow \mathrm{J} / \psi \pi^{+} \pi^{-}\right] \mathrm{pK} \mathrm{K}^{-}$have two additional pions to reconstruct compared to the reference mode $\Lambda_{\mathrm{b}}^{0} \rightarrow \mathrm{J} / \psi \mathrm{pK}^{-}$. The uncertainty associated with the reconstruction of these additional low- $p_{\mathrm{T}}$ tracks has two independent contributions. First, the uncertainties in the amount and distribution of material in the detector result in an uncertainty of $2.0 \%$ per additional final-state pion due to 
the modelling of hadron interactions [41]. Second, the small difference in the track finding efficiency between data and simulation is corrected using a data-driven technique [41]. The uncertainties in the correction factors are propagated to the efficiency ratios by means of pseudoexperiments. This results in a systematic uncertainty of $3.2 \%$ for the ratio $\left.R^{\psi(2 \mathrm{~S})}\right|_{\psi(2 \mathrm{~S}) \rightarrow \mathrm{J} / \psi \pi^{+} \pi^{-}}$and $2.7 \%$ for the ratio $R^{\mathrm{J} / \psi \pi^{+} \pi^{-}}$.

The systematic uncertainties related to the hadron identification efficiency, $0.1(0.2) \%$ for $R^{\psi(2 \mathrm{~S})}\left(R^{\mathrm{J} / \psi} \pi^{+} \pi^{-}\right)$ratios, reflect the limited sizes of the calibration samples, and are propagated to the ratios $R^{\psi(2 \mathrm{~S})}$ and $R^{\mathrm{J} / \psi \pi^{+} \pi^{-}}$by means of pseudoexperiments.

The trigger efficiency for events with $\mathrm{J} / \psi \rightarrow \mu^{+} \mu^{-}$and $\psi(2 \mathrm{~S}) \rightarrow \mu^{+} \mu^{-}$produced in beauty hadron decays is studied in data. A systematic uncertainty of $1.1 \%$ is assigned based on a comparison between data and simulation of the ratio of trigger efficiencies for high-yield samples of $\mathrm{B}^{+} \rightarrow \mathrm{J} / \psi \mathrm{K}^{+}$and $\mathrm{B}^{+} \rightarrow \psi(2 \mathrm{~S}) \mathrm{K}^{+}$decays [13].

Another source of uncertainty is the potential disagreement between data and simulation in the estimation of efficiencies, due to effects not considered above. This is studied by varying the selection criteria in ranges that lead to as much as $\pm 20 \%$ change in the measured signal yields. The stability is tested by comparing the efficiency-corrected yields within these variations. The largest deviations range between $0.2 \%$ and $0.9 \%$ and are taken as systematic uncertainties.

Finally, a systematic uncertainty due to the limited size of the simulation sample is assigned. With all the systematic uncertainties added in quadrature, the total is $2.0 \%$ for the ratio $\left.R^{\psi(2 \mathrm{~S})}\right|_{\psi(2 \mathrm{~S}) \rightarrow \mu^{+} \mu^{-}}, 6.4 \%$ for the ratio $\left.R^{\psi(2 \mathrm{~S})}\right|_{\psi(2 \mathrm{~S}) \rightarrow \mathrm{J} / \psi \pi^{+} \pi^{-}}$and $6.4 \%$ for the ratio $R^{\mathrm{J} / \psi} \pi^{+} \pi^{-}$.

\subsection{Results}

Using eq. (4.1) and the ratios of yields and efficiencies determined above, the ratio $R^{\psi(2 \mathrm{~S})}$ is measured for each $\psi(2 \mathrm{~S})$ decay mode separately:

$$
\begin{aligned}
\left.R^{\psi(2 \mathrm{~S})}\right|_{\psi(2 \mathrm{~S}) \rightarrow \mu^{+} \mu^{-}} & =(20.74 \pm 0.88 \pm 0.41 \pm 0.47) \times 10^{-2}, \\
\left.R^{\psi(2 \mathrm{~S})}\right|_{\psi(2 \mathrm{~S}) \rightarrow \mathrm{J} / \psi \pi^{+} \pi^{-}} & =(20.55 \pm 1.52 \pm 1.32 \pm 0.18) \times 10^{-2},
\end{aligned}
$$

where the first uncertainty is statistical, the second is systematic and the third is related to the uncertainties on the dielectron $\mathrm{J} / \psi$ and $\psi(2 \mathrm{~S})$ branching fractions and the $\psi(2 \mathrm{~S}) \rightarrow \mathrm{J} / \psi \pi^{+} \pi^{-}$branching fraction. The average of the ratios in eq. (4.3) is

$$
R^{\psi(2 \mathrm{~S})}=(20.70 \pm 0.76 \pm 0.46 \pm 0.37) \times 10^{-2} .
$$

In this average the systematic uncertainties related to the normalization channel, $\Lambda_{\mathrm{b}}^{0} \rightarrow \mathrm{J} / \psi \mathrm{pK}^{-}$, and the trigger efficiency are considered to be $100 \%$ correlated while other systematic uncertainties are treated as uncorrelated.

The ratio of the branching fractions of $\Lambda_{\mathrm{b}}^{0} \rightarrow \mathrm{J} / \psi \pi^{+} \pi^{-} \mathrm{pK}^{-}$and $\Lambda_{\mathrm{b}}^{0} \rightarrow \mathrm{J} / \psi \mathrm{pK} K^{-}$is found to be

$$
R^{\mathrm{J} / \psi \pi^{+} \pi^{-}}=(20.86 \pm 0.96 \pm 1.34) \times 10^{-2},
$$

where contributions via intermediate resonances are included. 
The absolute branching fractions $\Lambda_{\mathrm{b}}^{0} \rightarrow \psi(2 \mathrm{~S}) \mathrm{pK}^{-}$and $\Lambda_{\mathrm{b}}^{0} \rightarrow \mathrm{J} / \psi \pi^{+} \pi^{-} \mathrm{pK}^{-}$are derived using the branching fraction $\mathcal{B}\left(\Lambda_{\mathrm{b}}^{0} \rightarrow \mathrm{J} / \psi \mathrm{pK}^{-}\right)=\left(3.04 \pm 0.04 \pm 0.06 \pm 0.33_{-0.27}^{+0.43}\right) \times 10^{-4}$, measured in ref. [9], where the third uncertainty is due to the uncertainty on the branching fraction of the decay $\mathrm{B}^{0} \rightarrow \mathrm{J} / \psi \overline{\mathrm{K}}^{*}(892)^{0}$ and the fourth is due to the knowledge of the ratio of fragmentation fractions $f_{\Lambda_{\mathrm{b}}^{0}} / f_{\mathrm{d}}$. They are found to be

$$
\begin{aligned}
\mathcal{B}\left(\Lambda_{\mathrm{b}}^{0} \rightarrow \psi(2 \mathrm{~S}) \mathrm{pK}^{-}\right) & =\left(6.29 \pm 0.23 \pm 0.14_{-0.90}^{+1.14}\right) \times 10^{-5}, \\
\mathcal{B}\left(\Lambda_{\mathrm{b}}^{0} \rightarrow \mathrm{J} / \psi \pi^{+} \pi^{-} \mathrm{pK}^{-}\right) & =\left(6.34 \pm 0.29 \pm 0.41_{-0.91}^{+1.15}\right) \times 10^{-5},
\end{aligned}
$$

where the third uncertainty comes from the uncertainties in the branching fractions of $\Lambda_{\mathrm{b}}^{0} \rightarrow \mathrm{J} / \psi \mathrm{pK}^{-}, \psi(2 \mathrm{~S}) \rightarrow \mathrm{J} / \psi \pi^{+} \pi^{-}, \psi(2 \mathrm{~S}) \rightarrow \mathrm{e}^{+} \mathrm{e}^{-}$and $\mathrm{J} / \psi \rightarrow \mathrm{e}^{+} \mathrm{e}^{-}$decays.

From the two separate measurements of the ratio $R^{\psi(2 \mathrm{~S})}$ via different decay modes of the $\psi(2 \mathrm{~S})$ meson (eq. (4.3)), the ratio of the $\psi(2 \mathrm{~S}) \rightarrow \mu^{+} \mu^{-}$and $\psi(2 \mathrm{~S}) \rightarrow \mathrm{J} / \psi \pi^{+} \pi^{-}$ branching fractions is calculated as

$$
\begin{aligned}
\frac{\mathcal{B}\left(\psi(2 \mathrm{~S}) \rightarrow \mu^{+} \mu^{-}\right)}{\mathcal{B}\left(\psi(2 \mathrm{~S}) \rightarrow \mathrm{J} / \psi \pi^{+} \pi^{-}\right)} & =\frac{N_{\psi(2 \mathrm{~S}) \rightarrow \mu^{+} \mu^{-}}}{N_{\psi(2 \mathrm{~S}) \rightarrow \mathrm{J} / \psi \pi^{+} \pi^{-}}} \times \frac{\varepsilon_{\psi(2 \mathrm{~S}) \rightarrow \mathrm{J} / \psi \pi^{+} \pi^{-}}^{\Lambda_{\mathrm{b}}^{0}}}{\Lambda_{\mathrm{b}}^{0}} \times \mathcal{B}\left(\mathrm{J} / \psi \rightarrow \mu^{+} \mu^{-}\right) \\
& =(2.30 \pm 0.20 \pm 0.12 \pm 0.01) \times 1 \mu^{+} \mu^{-}
\end{aligned}
$$

where the third uncertainty is related to the uncertainty of the known branching fraction $\mathcal{B}\left(\mathrm{J} / \psi \rightarrow \mu^{+} \mu^{-}\right)=(5.961 \pm 0.033) \%$ [36]. This result is in agreement with the world average of $(2.29 \pm 0.25) \times 10^{-2}$ [36] based on results of the E672/E706 [44] and BaBar [45] collaborations, and has similar precision.

\section{$5 \quad$ Measurement of $\Lambda_{\mathrm{b}}^{0}$ baryon mass}

The low energy release in $\Lambda_{\mathrm{b}}^{0} \rightarrow \psi(2 \mathrm{~S}) \mathrm{pK}^{-}$and $\Lambda_{\mathrm{b}}^{0} \rightarrow \mathrm{J} / \psi \pi^{+} \pi^{-} \mathrm{pK}^{-}$decays allows the $\Lambda_{\mathrm{b}}^{0}$ mass to be determined with a small systematic uncertainty. The mass is measured using four decay channels: $\Lambda_{\mathrm{b}}^{0} \rightarrow \psi(2 \mathrm{~S})\left[\rightarrow \mu^{+} \mu^{-}\right] \mathrm{pK}^{-}$, $\Lambda_{\mathrm{b}}^{0} \rightarrow \psi(2 \mathrm{~S})\left[\rightarrow \mathrm{J} / \psi \pi^{+} \pi^{-}\right] \mathrm{pK}^{-}, \Lambda_{\mathrm{b}}^{0} \rightarrow \mathrm{J} / \psi \pi^{+} \pi^{-} \mathrm{pK}^{-}$and $\Lambda_{\mathrm{b}}^{0} \rightarrow \mathrm{J} / \psi \mathrm{pK}^{-}$. The mass distributions for the $\Lambda_{\mathrm{b}}^{0} \rightarrow \psi(2 \mathrm{~S})\left[\rightarrow \mu^{+} \mu^{-}\right] \mathrm{pK}^{-}$and $\Lambda_{\mathrm{b}}^{0} \rightarrow \mathrm{J} / \psi \mathrm{pK}^{-}$channels are shown in figure 1. In the $\Lambda_{\mathrm{b}}^{0} \rightarrow \psi(2 \mathrm{~S})\left[\rightarrow \mathrm{J} / \psi \pi^{+} \pi^{-}\right] \mathrm{pK} \mathrm{p}^{-}$channel, the $\mathrm{J} / \psi \pi^{+} \pi^{-}$system is constrained to the nominal $\psi(2 \mathrm{~S})$ mass [36] to improve the precision. In the $\Lambda_{\mathrm{b}}^{0} \rightarrow \mathrm{J} / \psi \pi^{+} \pi^{-} \mathrm{pK}^{-}$channel, to avoid overlap with the $\Lambda_{\mathrm{b}}^{0} \rightarrow \psi(2 \mathrm{~S})\left[\rightarrow \mathrm{J} / \psi \pi^{+} \pi^{-}\right] \mathrm{pK}^{-}$channel the $\psi(2 \mathrm{~S})$ region is vetoed, i.e. the mass of the $\mathrm{J} / \psi \pi^{+} \pi^{-}$combination is required to be outside the range $3670<m\left(\mathrm{~J} / \psi \pi^{+} \pi^{-}\right)<3700 \mathrm{MeV} / c^{2}$. The mass distributions for these two samples, along with the result of an unbinned extended maximum likelihood fit using the model described in section 4.1, are shown in figure 3 .

The systematic uncertainties on the measurement of the $\Lambda_{\mathrm{b}}^{0}$ baryon mass for all four channels are listed in table 4 . The precision of the absolute momentum scale calibration of $0.03 \%$ is the dominant source of uncertainty $[23,46]$. This uncertainty is proportional to the energy release in the decay and is minimal for the processes with a $\psi(2 S)$ in the final state. A further uncertainty is related to the energy loss in the material of the tracking 

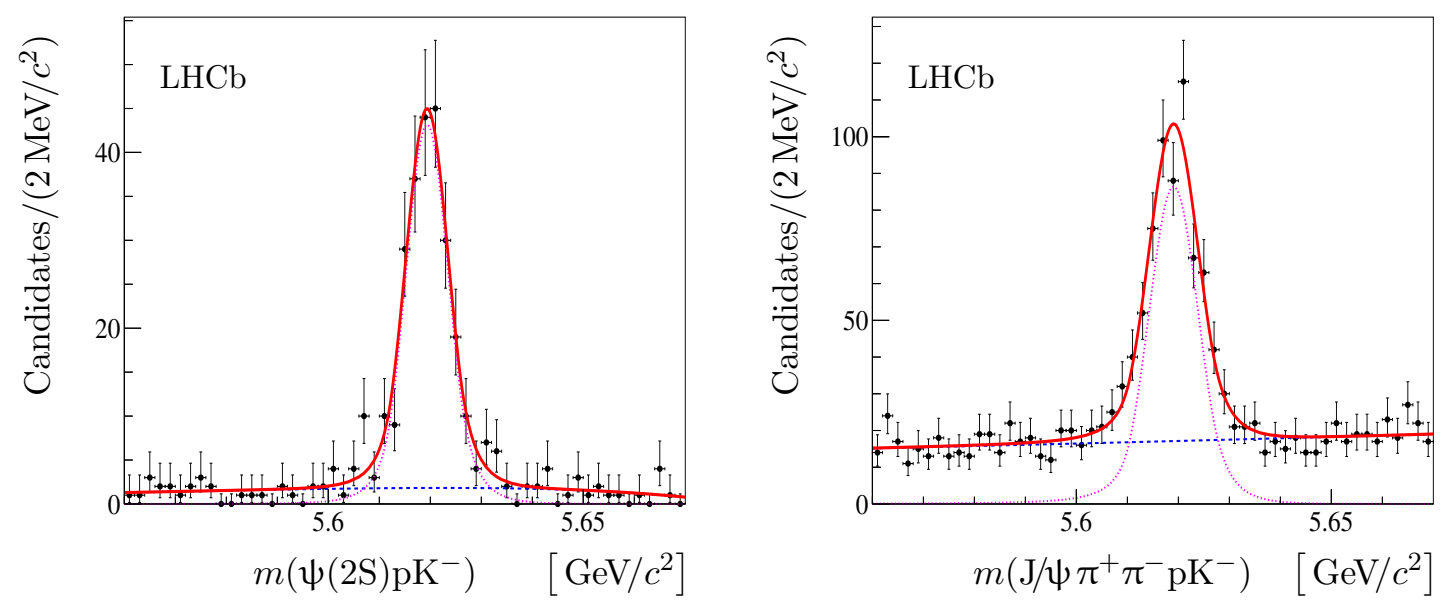

Figure 3. Left: mass distribution of selected $\Lambda_{\mathrm{b}}^{0} \rightarrow \psi(2 \mathrm{~S})\left[\rightarrow \mathrm{J} / \psi \pi^{+} \pi^{-}\right] \mathrm{pK} \mathrm{K}^{-}$candidates with an additional constraint for the $\psi(2 \mathrm{~S})$ mass [36]. Right: mass distribution of selected $\Lambda_{\mathrm{b}}^{0} \rightarrow \mathrm{J} / \psi \pi^{+} \pi^{-} \mathrm{pK}^{-}$candidates with a requirement of the $\mathrm{J} / \psi \pi^{+} \pi^{-}$combination mass to be outside the range $3670<m\left(\mathrm{~J} / \psi \pi^{+} \pi^{-}\right)<3700 \mathrm{MeV} / c^{2}$. The total fit function (solid red), the $\Lambda_{\mathrm{b}}^{0}$ signal contribution (dotted magenta) and the combinatorial background (dashed blue) are shown.

\begin{tabular}{|lcccc|}
\hline & $\mathrm{J} / \psi$ & $\psi(2 \mathrm{~S}) \rightarrow \mu^{+} \mu^{-}$ & $\psi(2 \mathrm{~S}) \rightarrow \mathrm{J} / \psi \pi^{+} \pi^{-}$ & $\mathrm{J} / \psi \pi^{+} \pi^{-}, \psi(2 \mathrm{~S})$ \\
\hline Momentum scale & 0.34 & 0.19 & 0.15 & 0.26 \\
Energy loss correction & 0.03 & 0.02 & 0.06 & 0.07 \\
Fit model & 0.04 & 0.03 & 0.08 & 0.05 \\
\hline Sum in quadrature & 0.34 & 0.19 & 0.18 & 0.27 \\
\hline
\end{tabular}

Table 4. Systematic uncertainties (in $\mathrm{MeV} / \mathrm{c}^{2}$ ) on the $\Lambda_{\mathrm{b}}^{0}$ mass using the decay modes $\quad \Lambda_{\mathrm{b}}^{0} \rightarrow \mathrm{J} / \psi \mathrm{pK}^{-}, \quad \Lambda_{\mathrm{b}}^{0} \rightarrow \psi(2 \mathrm{~S})\left[\rightarrow \mu^{+} \mu^{-}\right] \mathrm{pK}^{-}, \quad \Lambda_{\mathrm{b}}^{0} \rightarrow \psi(2 \mathrm{~S})\left[\rightarrow \mathrm{J} / \psi \pi^{+} \pi^{-}\right] \mathrm{pK}^{-} \quad$ and $\Lambda_{\mathrm{b}}^{0} \rightarrow \mathrm{J} / \psi \pi^{+} \pi^{-} \mathrm{pK}{ }^{-}$with the $\mathrm{J} / \psi \pi^{+} \pi^{-}$mass outside the $\psi(2 \mathrm{~S})$ region.

system [47], which is known with an accuracy of $10 \%$ [48]. This effect is estimated by varying the energy loss correction in the reconstruction by $10 \%$ and taking the observed mass shift as an uncertainty. The uncertainty due to the fit model is estimated using the same set of cross-check models for the signal and background parameterization as considered in section 4 , with the maximum deviation in the mass assigned as a systematic uncertainty. The uncertainties on the masses of the $\mathrm{J} / \psi$ and $\psi(2 \mathrm{~S})$ mesons [36] are small and are therefore neglected.

As a cross-check, the data sample is divided into four parts, for data collected at $\sqrt{s}=7$ and $8 \mathrm{TeV}$ and with different magnet polarities. The measured masses are consistent among these subsamples, and therefore no systematic uncertainty is assigned. To check the effect of the selection criteria (see section 3), the high-yield $\Lambda_{\mathrm{b}}^{0} \rightarrow \mathrm{J} / \psi \mathrm{pK}^{-}$decay channel is used. No sizeable dependence of the mass on the selection criteria is observed and no additional uncertainty is assigned.

The results from the four decay channels are presented in table 5. To combine them, correlations must be taken into account. The statistical uncertainties and those related 


\begin{tabular}{|lc|}
\hline Channel & $M\left(\Lambda_{\mathrm{b}}^{0}\right)\left[\mathrm{MeV} / \mathrm{c}^{2}\right]$ \\
\hline$\Lambda_{\mathrm{b}}^{0} \rightarrow \mathrm{J} / \psi \mathrm{pK}^{-}$ & $5619.62 \pm 0.04 \pm 0.34$ \\
$\Lambda_{\mathrm{b}}^{0} \rightarrow \psi(2 \mathrm{~S})\left[\rightarrow \mu^{+} \mu^{-}\right] \mathrm{pK}^{-}$ & $5619.84 \pm 0.18 \pm 0.19$ \\
$\Lambda_{\mathrm{b}}^{0} \rightarrow \psi(2 \mathrm{~S})\left[\rightarrow \mathrm{J} / \psi \pi^{+} \pi^{-}\right] \mathrm{pK}^{-}$ & $5619.38 \pm 0.33 \pm 0.18$ \\
$\Lambda_{\mathrm{b}}^{0} \rightarrow \mathrm{J} / \psi \pi^{+} \pi^{-} \mathrm{pK}^{-}$excluding $\psi(2 \mathrm{~S})$ & $5619.08 \pm 0.30 \pm 0.27$ \\
\hline
\end{tabular}

Table 5. Measured $\Lambda_{\mathrm{b}}^{0}$ mass in different decay channels. The first uncertainty is statistical and the second is systematic.

to the fit procedure are treated as uncorrelated while those due to the momentum scale and energy loss correction are considered to be fully correlated. The combined value of the $\Lambda_{\mathrm{b}}^{0}$ mass is

$$
M\left(\Lambda_{\mathrm{b}}^{0}\right)=5619.65 \pm 0.17 \pm 0.17 \mathrm{MeV} / c^{2},
$$

where the first uncertainty is statistical and the second systematic. The $\chi^{2} /$ ndf calculated for the individual measurements with respect to the combined value is $3.0 / 3$. This is the most precise measurement of any b-hadron mass reported to date.

Previous direct measurements of the $\Lambda_{\mathrm{b}}^{0}$ mass by $\mathrm{LHCb}$ were made using the decay $\Lambda_{\mathrm{b}}^{0} \rightarrow \mathrm{J} / \psi \Lambda^{0}[23,47]$ and are statistically independent of the results of this study. The combination obtained here is consistent with, and more precise than, the results of these earlier studies. The $\mathrm{LHCb}$ results are combined, taking the statistical uncertainties and those related to the fit procedure to be uncorrelated and those due to the energy loss correction to be fully correlated. The uncertainty due to the momentum scale in ref. [23] is also taken to be fully correlated, whereas in ref. [47] a different alignment and calibration procedure was used and so the corresponding uncertainty is considered to be uncorrelated with the other measurements. The result of the combination is dominated by the measurements of this analysis and is

$$
M\left(\Lambda_{\mathrm{b}}^{0}\right)=5619.65 \pm 0.16 \pm 0.14 \mathrm{MeV} / c^{2}
$$

where the uncertainties are statistical and systematic. The $\chi^{2} /$ ndf calculated for the individual measurements with respect to the combined value is $3.4 / 5$. The measured mass is in agreement with, but more precise than, the results of the ATLAS [49] and CDF [5] collaborations.

From the value of the $\Lambda_{\mathrm{b}}^{0}$ mass in eq. (5.2) and a precise measurement of the mass difference between the $\Lambda_{\mathrm{b}}^{0}$ and $\mathrm{B}^{0}$ hadrons reported in ref. [7], the mass of the $\mathrm{B}^{0}$ meson is calculated to be

$$
M\left(\mathrm{~B}^{0}\right)=5279.93 \pm 0.39 \mathrm{MeV} / c^{2},
$$

where the correlation of $41 \%$ between the $\mathrm{LHCb}$ measurements of the $\Lambda_{\mathrm{b}}^{0}$ mass and the $\Lambda_{\mathrm{b}}^{0}-\mathrm{B}^{0}$ mass splitting has been taken into account. This is in agreement with the current world average of $5279.61 \pm 0.16 \mathrm{MeV} / c^{2}[36]$. 


\section{Results and summary}

The $\Lambda_{\mathrm{b}}^{0} \rightarrow \psi(2 \mathrm{~S}) \mathrm{pK}^{-}$and $\Lambda_{\mathrm{b}}^{0} \rightarrow \mathrm{J} / \psi \pi^{+} \pi^{-} \mathrm{pK}^{-}$decay modes are observed using a sample of pp collisions at centre-of-mass energies of 7 and $8 \mathrm{TeV}$, corresponding to an integrated luminosity of $3 \mathrm{fb}^{-1}$. With results from the channels $\psi(2 \mathrm{~S}) \rightarrow \mu^{+} \mu^{-}$and $\psi(2 \mathrm{~S}) \rightarrow \mathrm{J} / \psi \pi^{+} \pi^{-}$combined, the ratio of branching fractions is measured:

$$
R^{\psi(2 \mathrm{~S})}=\frac{\mathcal{B}\left(\Lambda_{\mathrm{b}}^{0} \rightarrow \psi(2 \mathrm{~S}) \mathrm{pK}^{-}\right)}{\mathcal{B}\left(\Lambda_{\mathrm{b}}^{0} \rightarrow \mathrm{J} / \psi \mathrm{pK}^{-}\right)}=(20.70 \pm 0.76 \pm 0.46 \pm 0.37) \times 10^{-2},
$$

where the first uncertainty is statistical, the second is systematic and the third is related to the uncertainties of the known dielectron $\mathrm{J} / \psi$ and $\psi(2 \mathrm{~S})$ branching fractions and of the branching fraction of the $\psi(2 \mathrm{~S}) \rightarrow \mathrm{J} / \psi \pi^{+} \pi^{-}$decay. The ratio of branching fractions for $\Lambda_{\mathrm{b}}^{0} \rightarrow \mathrm{J} / \psi \pi^{+} \pi^{-} \mathrm{pK}^{-}$and $\Lambda_{\mathrm{b}}^{0} \rightarrow \mathrm{J} / \psi \mathrm{pK} \mathrm{p}^{-}$is

$$
R^{\mathrm{J} / \psi} \pi^{+} \pi^{-}=\frac{\mathcal{B}\left(\Lambda_{\mathrm{b}}^{0} \rightarrow \mathrm{J} / \psi \pi^{+} \pi^{-} \mathrm{pK}^{-}\right)}{\mathcal{B}\left(\Lambda_{\mathrm{b}}^{0} \rightarrow \mathrm{J} / \psi \mathrm{pK}^{-}\right)}=(20.86 \pm 0.96 \pm 1.34) \times 10^{-2},
$$

where the first uncertainty is statistical, the second is systematic and contributions via intermediate resonances are included.

From measurements of the ratio $R^{\psi(2 \mathrm{~S})}$ via two different decay modes of the $\psi(2 \mathrm{~S})$ meson it is determined that

$$
\frac{\mathcal{B}\left(\psi(2 \mathrm{~S}) \rightarrow \mu^{+} \mu^{-}\right)}{\mathcal{B}\left(\psi(2 \mathrm{~S}) \rightarrow \mathrm{J} / \psi \pi^{+} \pi^{-}\right)}=(2.30 \pm 0.20 \pm 0.12 \pm 0.01) \times 10^{-2},
$$

where the first uncertainty is statistical, the second is systematic and the third is related to the uncertainty on $\mathcal{B}\left(\mathrm{J} / \psi \rightarrow \mu^{+} \mu^{-}\right)$. This is the most precise direct measurement of this ratio to date.

Using $\Lambda_{\mathrm{b}}^{0} \rightarrow \psi(2 \mathrm{~S}) \mathrm{pK}^{-}, \Lambda_{\mathrm{b}}^{0} \rightarrow \mathrm{J} / \psi \pi^{+} \pi^{-} \mathrm{pK}^{-}$and $\Lambda_{\mathrm{b}}^{0} \rightarrow \mathrm{J} / \psi \mathrm{pK}^{-}$decays, the mass of the $\Lambda_{\mathrm{b}}^{0}$ baryon is measured to be

$$
M\left(\Lambda_{\mathrm{b}}^{0}\right)=5619.65 \pm 0.17 \pm 0.17 \mathrm{MeV} / c^{2},
$$

where the first uncertainty is statistical and the second is systematic. Combining this result with previous LHCb measurements that used the channel $\Lambda_{\mathrm{b}}^{0} \rightarrow \mathrm{J} / \psi \Lambda^{0}[23,47]$ gives

$$
M\left(\Lambda_{\mathrm{b}}^{0}\right)=5619.65 \pm 0.16 \pm 0.14 \mathrm{MeV} / c^{2},
$$

where the first uncertainty is statistical and the second is systematic. This is the most precise determination of the mass of any $b$ hadron to date.

\section{Acknowledgments}

We express our gratitude to our colleagues in the CERN accelerator departments for the excellent performance of the LHC. We thank the technical and administrative staff at the $\mathrm{LHCb}$ institutes. We acknowledge support from CERN and from 
the national agencies: CAPES, CNPq, FAPERJ and FINEP (Brazil); NSFC (China); CNRS/IN2P3 (France); BMBF, DFG and MPG(Germany); INFN(Italy); FOM and NWO (The Netherlands); MNiSW and NCN(Poland); MEN/IFA (Romania); MinES and FANO (Russia); MinECo (Spain); SNSF and SER (Switzerland); NASU (Ukraine); STFC (United Kingdom); NSF (U.S.A.). We acknowledge the computing resources that are provided by CERN, IN2P3 (France), KIT and DESY(Germany), INFN (Italy), SURF (The Netherlands), PIC (Spain), GridPP (United Kingdom), RRCKI and Yandex LLC (Russia), CSCS (Switzerland), IFIN-HH (Romania), CBPF (Brazil), PL-GRID (Poland) and OSC (U.S.A.). We are indebted to the communities behind the multiple open source software packages on which we depend. Individual groups or members have received support from AvH Foundation (Germany), EPLANET, Marie Skłodowska-Curie Actions and ERC(European Union), Conseil Général de Haute-Savoie, Labex ENIGMASS and OCEVU, Région Auvergne (France), RFBR and Yandex LLC(Russia), GVA, XuntaGal and GENCAT (Spain), Herchel Smith Fund, The Royal Society, Royal Commission for the Exhibition of 1851 and the Leverhulme Trust (United Kingdom).

Open Access. This article is distributed under the terms of the Creative Commons Attribution License (CC-BY 4.0), which permits any use, distribution and reproduction in any medium, provided the original author(s) and source are credited.

\section{References}

[1] M. Neubert, Heavy quark symmetry, Phys. Rept. 245 (1994) 259 [hep-ph/9306320] [INSPIRE].

[2] A.V. Manohar and M.B. Wise, Heavy quark physics, Camb. Monogr. Part. Phys. Nucl. Phys. Cosmol., volume 10, Camrbidge University Press, Cambridge U.K. (2000).

[3] CDF collaboration, A. Abulencia et al., Measurement of $\sigma\left(\Lambda_{b}^{0}\right) / \sigma\left(\bar{B}^{0}\right) \times \operatorname{BR}\left(\Lambda_{b}^{0} \rightarrow \Lambda_{c}^{+} \pi^{-}\right) / \operatorname{BR}\left(\bar{B}^{0} \rightarrow D^{+} \pi^{-}\right)$in pp collisions at $\sqrt{s}=1.96 \mathrm{TeV}$, Phys. Rev. Lett. 98 (2007) 122002 [hep-ex/0601003] [inSPIRE].

[4] D0 collaboration, V.M. Abazov et al., Measurement of the production fraction times branching fraction $f\left(b \rightarrow \Lambda_{b}\right) \cdot \mathcal{B}\left(\Lambda_{b} \rightarrow J / \psi \Lambda\right)$, Phys. Rev. D 84 (2011) 031102 [arXiv: 1105.0690] [INSPIRE].

[5] CDF collaboration, T.A. Aaltonen et al., Mass and lifetime measurements of bottom and charm baryons in p p collisions at $\sqrt{s}=1.96$ TeV, Phys. Rev. D 89 (2014) 072014 [arXiv: 1403.8126] [INSPIRE].

[6] LHCb collaboration, Studies of beauty baryon decays to $D^{0} p h^{-}$and $\Lambda_{c}^{+} h^{-}$final states, Phys. Rev. D 89 (2014) 032001 [arXiv: 1311.4823] [INSPIRE].

[7] LHCb collaboration, Study of beauty hadron decays into pairs of charm hadrons, Phys. Rev. Lett. 112 (2014) 202001 [arXiv: 1403.3606] [INSPIRE].

[8] LHCb collaboration, Observation of the $\Lambda_{b}^{0} \rightarrow J / \psi p \pi^{-}$decay, JHEP 07 (2014) 103 [arXiv: 1406.0755] [INSPIRE]. 
[9] LHCb collaboration, Study of the production of $\Lambda_{b}^{0}$ and $\bar{B}^{0}$ hadrons in pp collisions and first measurement of the $\Lambda_{b}^{0} \rightarrow J / \psi p K^{-}$branching fraction, Chin. Phys. C 40 (2016) 011001 [arXiv: 1509.00292] [INSPIRE].

[10] LHCb collaboration, Precision measurement of the ratio of the $\Lambda_{b}^{0}$ to $\bar{B}^{0}$ lifetimes, Phys. Lett. B 734 (2014) 122 [arXiv: 1402.6242] [INSPIRE].

[11] LHCb collaboration, Precision measurement of the mass and lifetime of the $\Xi_{b}^{-}$baryon, Phys. Rev. Lett. 113 (2014) 242002 [arXiv:1409.8568] [INSPIRE].

[12] LHCb collaboration, Observation of $J / \psi p$ resonances consistent with pentaquark states in $\Lambda_{b}^{0} \rightarrow J / \psi K^{-} p$ decays, Phys. Rev. Lett. 115 (2015) 072001 [arXiv:1507.03414] [inSPIRE].

[13] LHCb collaboration, Measurement of relative branching fractions of $B$ decays to $\psi(2 S)$ and J $\psi \psi$ mesons, Eur. Phys. J. C 72 (2012) 2118 [arXiv:1205.0918] [InSPIRE].

[14] LHCb collaboration, Observations of $B_{s}^{0} \rightarrow \psi(2 S) \eta$ and $B_{(s)}^{0} \rightarrow \psi(2 S) \pi^{+} \pi^{-}$decays, Nucl. Phys. B 871 (2013) 403 [arXiv:1302.6354] [INSPIRE].

[15] LHCb collaboration, Observation of $B_{s}^{0} \rightarrow \chi_{c 1} \phi$ decay and study of $B^{0} \rightarrow \chi_{c 1,2} K^{* 0}$ decays, Nucl. Phys. B 874 (2013) 663 [arXiv:1305.6511] [INSPIRE].

[16] LHCb collaboration, Measurement of $B_{c}^{+}$production in proton-proton collisions at $\sqrt{s}=8$ TeV, Phys. Rev. Lett. 114 (2015) 132001 [arXiv:1411.2943] [INSPIRE].

[17] LHCb collaboration, Study of $\eta-\eta^{\prime}$ mixing from measurement of $B_{(s)}^{0} \rightarrow J / \psi \eta^{(\prime)}$ decay rates, JHEP 01 (2015) 024 [arXiv: 1411.0943] [INSPIRE].

[18] LHCb collaboration, Measurement of the branching fraction ratio $\mathcal{B}\left(B_{c}^{+} \rightarrow \psi(2 S) \pi^{+}\right) / \mathcal{B}\left(B_{c}^{+} \rightarrow J / \psi \pi^{+}\right)$, Phys. Rev. D 92 (2015) 072007 [arXiv:1507.03516] [INSPIRE].

[19] ATLAS collaboration, Measurement of the branching ratio $\Gamma\left(\Lambda_{b}^{0} \rightarrow \psi(2 S) \Lambda^{0}\right) / \Gamma\left(\Lambda_{b}^{0} \rightarrow J / \psi \Lambda^{0}\right)$ with the ATLAS detector, Phys. Lett. B 751 (2015) 63 [arXiv: 1507.08202] [INSPIRE].

[20] LHCb collaboration, The LHCb detector at the LHC, 2008 JINST 3 S08005 [INSPIRE].

[21] LHCb collaboration, LHCb detector performance, Int. J. Mod. Phys. A 30 (2015) 1530022 [arXiv: 1412.6352] [INSPIRE].

[22] R. Aaij et al., Performance of the LHCb vertex locator, 2014 JINST 909007 [arXiv: 1405.7808] [INSPIRE].

[23] LHCb collaboration, Measurement of the $\Lambda_{b}^{0}, \Xi_{b}^{-}$and $\Omega_{b}^{-}$baryon masses, Phys. Rev. Lett. 110 (2013) 182001 [arXiv: 1302.1072] [INSPIRE].

[24] R. Aaij et al., The LHCb trigger and its performance in 2011, 2013 JINST 8 P04022 [arXiv: 1211.3055] [INSPIRE].

[25] T. Sjöstrand, S. Mrenna and P.Z. Skands, PYTHIA 6.4 physics and manual, JHEP 05 (2006) 026 [hep-ph/0603175] [INSPIRE].

[26] T. Sjöstrand, S. Mrenna and P.Z. Skands, A brief introduction to PYTHIA 8.1, Comput. Phys. Commun. 178 (2008) 852 [arXiv:0710.3820] [INSPIRE].

[27] LHCb collaboration, Handling of the generation of primary events in Gauss, the LHCb simulation framework, J. Phys. Conf. Ser. 331 (2011) 032047 [InSPIRE]. 
[28] D.J. Lange, The EvtGen particle decay simulation package, Nucl. Instrum. Meth. A 462 (2001) 152 [INSPIRE].

[29] P. Golonka and Z. Was, PHOTOS Monte Carlo: a precision tool for QED corrections in Z and $W$ decays, Eur. Phys. J. C 45 (2006) 97 [hep-ph/0506026] [INSPIRE].

[30] Geant4 collaboration, J. Allison et al., GEANT4 developments and applications, IEEE Trans. Nucl. Sci. 53 (2006) 270.

[31] GEANT4 collaboration, S. Agostinelli et al., GEANT4: a simulation toolkit, Nucl. Instrum. Meth. A 506 (2003) 250 [INSPIRE].

[32] LHCb collaboration, The LHCb simulation application, Gauss: design, evolution and experience, J. Phys. Conf. Ser. 331 (2011) 032023 [inSPIRE].

[33] LHCb collaboration, Evidence for the decay $B_{c}^{+} \rightarrow J / \psi 3 \pi^{+} 2 \pi^{-}$, JHEP 05 (2014) 148 [arXiv: 1404.0287] [INSPIRE].

[34] LHCB RICH Group collaboration, M. Adinolfi et al., Performance of the LHCb RICH detector at the LHC, Eur. Phys. J. C 73 (2013) 2431 [arXiv:1211.6759] [INSPIRE].

[35] F. Archilli et al., Performance of the muon identification at LHCb, 2013 JINST 8 P10020 [arXiv: 1306.0249] [INSPIRE].

[36] Particle Data Group collaboration, K.A. Olive et al., Review of particle physics, Chin. Phys. C 38 (2014) 090001 [inSPIRE].

[37] W.D. Hulsbergen, Decay chain fitting with a Kalman filter, Nucl. Instrum. Meth. A 552 (2005) 566 [physics/0503191] [INSPIRE].

[38] T. Skwarnicki, A study of the radiative cascade transitions between the $\Upsilon^{\prime}$ and $\Upsilon$ resonances, Ph.D. thesis, Institute of Nuclear Physics, Krakow, Poland (1986), DESY-F31-86-02 [INSPIRE].

[39] LHCb collaboration, Observation of $J / \psi$ pair production in pp collisions at $\sqrt{s}=7 \mathrm{TeV}$, Phys. Lett. B 707 (2012) 52 [arXiv:1109.0963] [inSPIRE].

[40] M. Pivk and F.R. Le Diberder, SPlot: a statistical tool to unfold data distributions, Nucl. Instrum. Meth. A 555 (2005) 356 [physics/0402083] [INSPIRE].

[41] LHCb collaboration, Measurement of the track reconstruction efficiency at LHCb, 2015 JINST 10 P02007 [arXiv: 1408.1251] [INSPIRE].

[42] BABAR collaboration, J.P. Lees et al., Branching fraction measurements of the color-suppressed decays $\bar{B}^{0} \rightarrow D^{(*) 0} \pi^{0}, D^{(*) 0} \eta, D^{(*) 0} \omega$ and $D^{(*) 0} \eta^{\prime}$ and measurement of the polarization in the decay $\bar{B}^{0} \rightarrow D^{* 0} \omega$, Phys. Rev. D 84 (2011) 112007 [arXiv:1107.5751] [INSPIRE].

[43] D. Martínez Santos and F. Dupertuis, Mass distributions marginalized over per-event errors, Nucl. Instrum. Meth. A 764 (2014) 150 [arXiv:1312.5000] [INSPIRE].

[44] E672 and E706 collaborations, A. Gribushin et al., Production of $J / \psi$ and $\psi(2 S)$ mesons in $\pi^{-}$Be collisions at $515 \mathrm{GeV} / \mathrm{c}$, FERMILAB-PUB-95-298 (1995).

[45] BABAR collaboration, B. Aubert et al., Measurement of the branching fractions for $\psi(2 S) \rightarrow e^{+} e^{-}$and $\psi(2 S) \rightarrow \mu^{+} \mu^{-}$, Phys. Rev. D 65 (2002) 031101 [hep-ex/0109004] [INSPIRE].

[46] LHCb collaboration, Precision measurement of D meson mass differences, JHEP 06 (2013) 065 [arXiv: 1304.6865] [INSPIRE]. 
[47] LHCb collaboration, Measurement of b-hadron masses, Phys. Lett. B 708 (2012) 241 [arXiv: 1112.4896] [INSPIRE].

[48] LHCb collaboration, Prompt $K_{s}^{0}$ production in pp collisions at $\sqrt{s}=0.9 \mathrm{TeV}$, Phys. Lett. B 693 (2010) 69 [arXiv: 1008.3105] [INSPIRE].

[49] ATLAS collaboration, Measurement of the $\Lambda_{b}^{0}$ lifetime and mass in the ATLAS experiment, Phys. Rev. D 87 (2013) 032002 [arXiv: 1207.2284] [INSPIRE]. 


\section{The LHCb collaboration}

R. Aaij ${ }^{39}$, C. Abellán Beteta ${ }^{41}$, B. Adeva ${ }^{38}$, M. Adinolfi ${ }^{47}$, A. Affolder ${ }^{53}$, Z. Ajaltouni ${ }^{5}$, S. Akar $^{6}$, J. Albrecht ${ }^{10}$, F. Alessio ${ }^{39}$, M. Alexander ${ }^{52}$, S. Ali ${ }^{42}$, G. Alkhazov ${ }^{31}$, P. Alvarez Cartelle ${ }^{54}$, A.A. Alves Jr ${ }^{58}$, S. Amato ${ }^{2}$, S. Amerio ${ }^{23}$, Y. Amhis ${ }^{7}$, L. An $^{3,40}$, L. Anderlini ${ }^{18}$, G. Andreassi ${ }^{40}$, M. Andreotti ${ }^{17, g}$, J.E. Andrews ${ }^{59}$, R.B. Appleby ${ }^{55}$, O. Aquines Gutierrez ${ }^{11}$, F. Archilli ${ }^{39}$, P. d'Argent ${ }^{12}$, A. Artamonov ${ }^{36}$, M. Artuso ${ }^{60}$, E. Aslanides ${ }^{6}$, G. Auriemma ${ }^{26, n}$, M. Baalouch ${ }^{5}$, S. Bachmann ${ }^{12}$, J.J. Back ${ }^{49}$, A. Badalov ${ }^{37}$, C. Baesso ${ }^{61}$, W. Baldini ${ }^{17,39}$, R.J. Barlow ${ }^{55}$, C. Barschel ${ }^{39}$, S. Barsuk ${ }^{7}$, W. Barter ${ }^{39}$, V. Batozskaya ${ }^{29}$, V. Battista ${ }^{40}$, A. Bay ${ }^{40}$, L. Beaucourt ${ }^{4}$, J. Beddow ${ }^{52}$, F. Bedeschi ${ }^{24}$, I. Bediaga ${ }^{1}$, L.J. Bel ${ }^{42}$, V. Bellee ${ }^{40}$, N. Belloli ${ }^{21, k}$, I. Belyaev ${ }^{32}$, E. Ben-Haim ${ }^{8}$, G. Bencivenni ${ }^{19}$, S. Benson ${ }^{39}$, J. Benton ${ }^{47}$, A. Berezhnoy ${ }^{33}$, R. Bernet ${ }^{41}$, A. Bertolin ${ }^{23}$, F. Betti ${ }^{15}$, M.-O. Bettler ${ }^{39}$, M. van Beuzekom ${ }^{42}$, S. Bifani ${ }^{46}$, P. Billoir ${ }^{8}$, T. Bird ${ }^{55}$, A. Birnkraut ${ }^{10}$, A. Bizzeti ${ }^{18, i}$, T. Blake ${ }^{49}$, F. Blanc ${ }^{40}$, J. Blouw ${ }^{11}$, S. Blusk ${ }^{60}$, V. Bocci ${ }^{26}$,

A. Bondar ${ }^{35}$, N. Bondar ${ }^{31,39}$, W. Bonivento ${ }^{16}$, A. Borgheresi ${ }^{21, k}$, S. Borghi ${ }^{55}$, M. Borisyak ${ }^{67}$, M. Borsato ${ }^{38}$, T.J.V. Bowcock ${ }^{53}$, E. Bowen ${ }^{41}$, C. Bozzi ${ }^{17,39}$, S. Braun ${ }^{12}$, M. Britsch ${ }^{12}$, T. Britton ${ }^{60}$, J. Brodzicka ${ }^{55}$, N.H. Brook ${ }^{47}$, E. Buchanan ${ }^{47}$, C. Burr ${ }^{55}$, A. Bursche ${ }^{2}$, J. Buytaert ${ }^{39}$, S. Cadeddu ${ }^{16}$, R. Calabrese ${ }^{17, g}$, M. Calvi ${ }^{21, k}$, M. Calvo Gomez ${ }^{37, p}$, P. Campana ${ }^{19}$, D. Campora Perez ${ }^{39}$, L. Capriotti ${ }^{55}$, A. Carbone ${ }^{15, e}$, G. Carboni ${ }^{25, l}$, R. Cardinale Ca,j $^{20}$

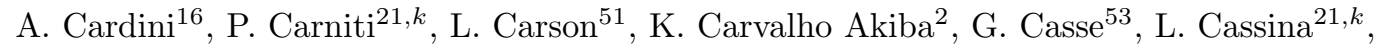
L. Castillo Garcia ${ }^{40}$, M. Cattaneo ${ }^{39}$, Ch. Cauet ${ }^{10}$, G. Cavallero ${ }^{20}$, R. Cenci ${ }^{24, t}$, M. Charles ${ }^{8}$, Ph. Charpentier ${ }^{39}$, G. Chatzikonstantinidis ${ }^{46}$, M. Chefdeville ${ }^{4}$, S. Chen ${ }^{55}$, S.-F. Cheung ${ }^{56}$, N. Chiapolini ${ }^{41}$, M. Chrzaszcz ${ }^{41,27}$, X. Cid Vidal ${ }^{39}$, G. Ciezarek ${ }^{42}$, P.E.L. Clarke ${ }^{51}$, M. Clemencic ${ }^{39}$, H.V. Cliff ${ }^{48}$, J. Closier ${ }^{39}$, V. Coco ${ }^{39}$, J. Cogan ${ }^{6}$, E. $\operatorname{Cogneras}^{5}$, V. Cogoni ${ }^{16, f}$, L. Cojocariu ${ }^{30}$, G. Collazuol ${ }^{23, r}$, P. Collins ${ }^{39}$, A. Comerma-Montells ${ }^{12}$, A. Contu ${ }^{39}$, A. Cook ${ }^{47}$, M. Coombes ${ }^{47}$, S. Coquereau ${ }^{8}$, G. Corti ${ }^{39}$, M. Corvo ${ }^{17, g}$, B. Couturier ${ }^{39}$, G.A. Cowan ${ }^{51}$, D.C. Craik $^{51}$, A. Crocombe ${ }^{49}$, M. Cruz Torres ${ }^{61}$, S. Cunliffe ${ }^{54}$, R. Currie ${ }^{54}$, C. D'Ambrosio ${ }^{39}$, E. Dall'Occo ${ }^{42}$, J. Dalseno ${ }^{47}$, P.N.Y. David ${ }^{42}$, A. Davis ${ }^{58}$, O. De Aguiar Francisco ${ }^{2}$, K. De Bruyn ${ }^{6}$, S. De Capua ${ }^{55}$, M. De Cian ${ }^{12}$, J.M. De Miranda ${ }^{1}$, L. De Paula ${ }^{2}$, P. De Simone ${ }^{19}$, C.-T. Dean ${ }^{52}$, D. Decamp ${ }^{4}$, M. Deckenhoff ${ }^{10}$, L. Del Buono ${ }^{8}$, N. Déléage ${ }^{4}$, M. Demmer ${ }^{10}$, D. Derkach ${ }^{67}$, O. Deschamps ${ }^{5}$, F. Dettori ${ }^{39}$, B. Dey ${ }^{22}$, A. Di Canto ${ }^{39}$, F. Di Ruscio ${ }^{25}$, H. Dijkstra ${ }^{39}$, S. Donleavy ${ }^{53}$, F. Dordei ${ }^{39}$, M. Dorigo $^{40}$, A. Dosil Suárez ${ }^{38}$, A. Dovbnya ${ }^{44}$, K. Dreimanis ${ }^{53}$, L. Dufour ${ }^{42}$, G. Dujany ${ }^{55}$, K. Dungs ${ }^{39}$, P. Durante ${ }^{39}$, R. Dzhelyadin ${ }^{36}$, A. Dziurda ${ }^{27}$, A. Dzyuba ${ }^{31}$, S. Easo ${ }^{50,39}$, U. Egede ${ }^{54}$, V. Egorychev ${ }^{32}$, S. Eidelman $^{35}$, S. Eisenhardt ${ }^{51}$, U. Eitschberger ${ }^{10}$, R. Ekelhof ${ }^{10}$, L. Eklund ${ }^{52}$, I. El Rifai ${ }^{5}$, Ch. Elsasser ${ }^{41}$, S. Ely ${ }^{60}$, S. Esen ${ }^{12}$, H.M. Evans ${ }^{48}$, T. Evans ${ }^{56}$, A. Falabella ${ }^{15}$, C. Färber ${ }^{39}$, N. Farley ${ }^{46}$, S. Farry ${ }^{53}$, R. Fay ${ }^{53}$, D. Fazzini ${ }^{21, k}$, D. Ferguson ${ }^{51}$, V. Fernandez Albor ${ }^{38}$, F. Ferrari ${ }^{15}$, F. Ferreira Rodrigues ${ }^{1}$, M. Ferro-Luzzi ${ }^{39}$, S. Filippov ${ }^{34}$, M. Fiore ${ }^{17,39, g}$, M. Fiorini ${ }^{17, g}$, M. Firlej ${ }^{28}$, C. Fitzpatrick ${ }^{40}$, T. Fiutowski ${ }^{28}$, F. Fleuret ${ }^{7, b}$, K. Fohl $^{39}$, M. Fontana ${ }^{16}$, F. Fontanelli ${ }^{20, j}$, D. C. Forshaw ${ }^{60}$, R. Forty ${ }^{39}$, M. Frank ${ }^{39}$, C. Frei ${ }^{39}$, M. Frosini ${ }^{18}$, J. Fu ${ }^{22}$, E. Furfaro ${ }^{25, l}$, A. Gallas Torreira ${ }^{38}$, D. Galli ${ }^{15, e}$, S. Gallorini ${ }^{23}$, S. Gambetta ${ }^{51}$, M. Gandelman ${ }^{2}$, P. Gandini ${ }^{56}$, Y. Gao ${ }^{3}$, J. García Pardiñas ${ }^{38}$, J. Garra Tico ${ }^{48}$, L. Garrido ${ }^{37}$, D. Gascon ${ }^{37}$, C. Gaspar $^{39}$, L. Gavardi ${ }^{10}$, G. Gazzoni ${ }^{5}$, D. Gerick ${ }^{12}$, E. Gersabeck ${ }^{12}$, M. Gersabeck ${ }^{55}$, T. Gershon ${ }^{49}$, Ph. Ghez ${ }^{4}$, S. Gianì ${ }^{40}$, V. Gibson ${ }^{48}$, O.G. Girard ${ }^{40}$, L. Giubega ${ }^{30}$, V.V. Gligorov ${ }^{39}$, C. Göbel ${ }^{61}$, D. Golubkov ${ }^{32}$, A. Golutvin ${ }^{54,39}$, A. Gomes ${ }^{1, a}$, C. Gotti ${ }^{21, k}$, M. Grabalosa Gándara ${ }^{5}$, R. Graciani Diaz ${ }^{37}$, L.A. Granado Cardoso ${ }^{39}$, E. Graugés ${ }^{37}$, E. Graverini ${ }^{41}$, G. Graziani ${ }^{18}$, A. Grecu ${ }^{30}$, P. Griffith ${ }^{46}$, L. Grillo ${ }^{12}$, O. Grünberg ${ }^{65}$, B. Gui ${ }^{60}$, E. Gushchin ${ }^{34}$, Yu. Guz ${ }^{36,39}$, T. Gys ${ }^{39}$, T. Hadavizadeh ${ }^{56}$, C. Hadjivasiliou ${ }^{60}$, G. Haefeli ${ }^{40}$, C. Haen ${ }^{39}$, S.C. Haines ${ }^{48}$, S. Hall ${ }^{54}$, B. Hamilton ${ }^{59}$, X. Han ${ }^{12}$, S. Hansmann-Menzemer ${ }^{12}$, N. Harnew ${ }^{56}$, S.T. Harnew ${ }^{47}$, J. Harrison ${ }^{55}$, 


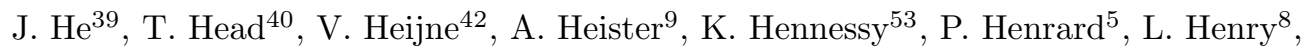
J.A. Hernando Morata ${ }^{38}$, E. van Herwijnen ${ }^{39}$, M. Heß ${ }^{65}$, A. Hicheur ${ }^{2}$, D. Hill ${ }^{56}$, M. Hoballah ${ }^{5}$, C. Hombach ${ }^{55}$, L. Hongming ${ }^{40}$, W. Hulsbergen ${ }^{42}$, T. Humair ${ }^{54}$, M. Hushchyn ${ }^{67}$, N. Hussain ${ }^{56}$, D. Hutchcroft ${ }^{53}$, M. Idzik ${ }^{28}$, P. Ilten ${ }^{57}$, R. Jacobsson ${ }^{39}$, A. Jaeger ${ }^{12}$, J. Jalocha ${ }^{56}$, E. Jans ${ }^{42}$, A. Jawahery ${ }^{59}$, M. John ${ }^{56}$, D. Johnson ${ }^{39}$, C.R. Jones ${ }^{48}$, C. Joram ${ }^{39}$, B. Jost ${ }^{39}$, N. Jurik ${ }^{60}$, S. Kandybei ${ }^{44}$, W. Kanso ${ }^{6}$, M. Karacson ${ }^{39}$, T.M. Karbach ${ }^{39, \dagger}$, S. Karodia ${ }^{52}$, M. Kecke ${ }^{12}$, M. Kelsey ${ }^{60}$, I.R. Kenyon ${ }^{46}$, M. Kenzie ${ }^{39}$, T. Ketel ${ }^{43}$, E. Khairullin ${ }^{67}$, B. Khanji ${ }^{21,39, k}$, C. Khurewathanakul ${ }^{40}$, T. Kirn ${ }^{9}$, S. Klaver ${ }^{55}$, K. Klimaszewski ${ }^{29}$, O. Kochebina ${ }^{7}$, M. Kolpin ${ }^{12}$, I. Komarov ${ }^{40}$, R.F. Koopman ${ }^{43}$, P. Koppenburg ${ }^{42,39}$, M. Kozeiha ${ }^{5}$, L. Kravchuk ${ }^{34}$, K. Kreplin ${ }^{12}$, M. Kreps ${ }^{49}$, P. Krokovny ${ }^{35}$, F. Kruse ${ }^{10}$, W. Krzemien ${ }^{29}$, W. Kucewicz ${ }^{27, o}$, M. Kucharczyk ${ }^{27}$, V. Kudryavtsev ${ }^{35}$, A. K. Kuonen ${ }^{40}$, K. Kurek ${ }^{29}$, T. Kvaratskheliya ${ }^{32}$, D. Lacarrere $^{39}$, G. Lafferty ${ }^{55,39}$, A. Lai ${ }^{16}$, D. Lambert ${ }^{51}$, G. Lanfranchi ${ }^{19}$, C. Langenbruch ${ }^{49}$, B. Langhans ${ }^{39}$, T. Latham ${ }^{49}$, C. Lazzeroni ${ }^{46}$, R. Le Gac ${ }^{6}$, J. van Leerdam ${ }^{42}$, J.-P. Lees ${ }^{4}$, R. Lefèvre ${ }^{5}$, A. Leflat ${ }^{33,39}$, J. Lefrançois ${ }^{7}$, E. Lemos $\mathrm{Cid}^{38}$, O. Leroy ${ }^{6}$, T. Lesiak ${ }^{27}$, B. Leverington ${ }^{12}, \mathrm{Y}^{3} \mathrm{Li}^{7}$,

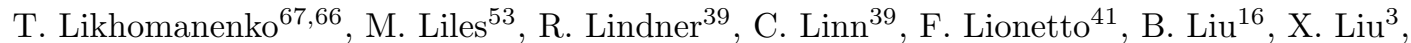
D. Loh ${ }^{49}$, I. Longstaff ${ }^{52}$, J.H. Lopes ${ }^{2}$, D. Lucchesi ${ }^{23, r}$, M. Lucio Martinez ${ }^{38}$, H. Luo ${ }^{51}$,

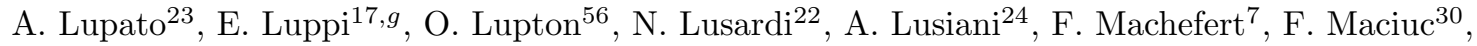
O. Maev ${ }^{31}$, K. Maguire ${ }^{55}$, S. Malde ${ }^{56}$, A. Malinin ${ }^{66}$, G. Manca ${ }^{7}$, G. Mancinelli ${ }^{6}$, P. Manning ${ }^{60}$, A. Mapelli ${ }^{39}$, J. Maratas ${ }^{5}$, J.F. Marchand ${ }^{4}$, U. Marconi ${ }^{15}$, C. Marin Benito ${ }^{37}$, P. Marino ${ }^{24,39, t}$, J. Marks ${ }^{12}$, G. Martellotti ${ }^{26}$, M. Martin ${ }^{6}$, M. Martinellii ${ }^{40}$, D. Martinez Santos ${ }^{38}$, F. Martinez Vidal ${ }^{68}$, D. Martins Tostes ${ }^{2}$, L.M. Massacrier ${ }^{7}$, A. Massafferri ${ }^{1}$, R. Matev ${ }^{39}$,

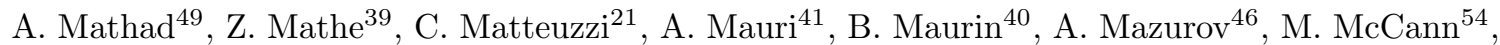
J. McCarthy ${ }^{46}$, A. McNab ${ }^{55}$, R. McNulty ${ }^{13}$, B. Meadows ${ }^{58}$, F. Meier ${ }^{10}$, M. Meissner ${ }^{12}$, D. Melnychuk ${ }^{29}$, M. Merk ${ }^{42}$, A Merli $22, u$, E Michielin ${ }^{23}$, D.A. Milanes ${ }^{64}$, M.-N. Minard ${ }^{4}$, D.S. Mitzel ${ }^{12}$, J. Molina Rodriguez ${ }^{61}$, I.A. Monroy ${ }^{64}$, S. Monteil $^{5}$, M. Morandin ${ }^{23}$, P. Morawski ${ }^{28}$,

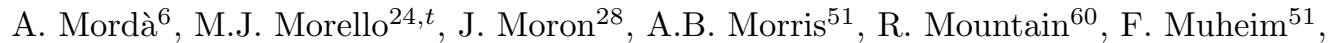
D. Müller ${ }^{55}$, J. Müller ${ }^{10}$, K. Müller ${ }^{41}$, V. Müller ${ }^{10}$, M. Mussini ${ }^{15}$, B. Muster ${ }^{40}$, P. Naik ${ }^{47}$,

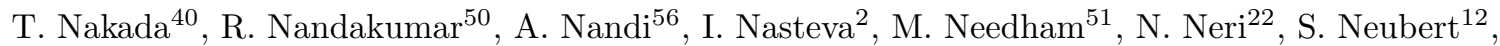
N. Neufeld ${ }^{39}$, M. Neuner ${ }^{12}$, A.D. Nguyen ${ }^{40}$, C. Nguyen-Mau ${ }^{40, q}$, V. Niess ${ }^{5}$, S. Nieswand ${ }^{9}$, R. $\mathrm{Niet}^{10}$, N. Nikitin ${ }^{33}$, T. Nikodem ${ }^{12}$, A. Novoselov ${ }^{36}$, D.P. O'Hanlon ${ }^{49}$, A. Oblakowska-Mucha ${ }^{28}$, V. Obraztsov ${ }^{36}$, S. Ogilvy ${ }^{52}$, O. Okhrimenko ${ }^{45}$, R. Oldeman ${ }^{16,48, f}$, C.J.G. Onderwater ${ }^{69}$, B. Osorio Rodrigues ${ }^{1}$, J.M. Otalora Goicochea ${ }^{2}$, A. Otto ${ }^{39}$, P. Owen ${ }^{54}$, A. Oyanguren $^{68}$, A. Palano ${ }^{14, d}$, F. Palombo ${ }^{22, u}$, M. Palutan ${ }^{19}$, J. Panman ${ }^{39}$, A. Papanestis ${ }^{50}$, M. Pappagallo ${ }^{52}$, L.L. Pappalardo ${ }^{17, g}$, C. Pappenheimer ${ }^{58}$, W. Parker $^{59}$, C. Parkes ${ }^{55}$, G. Passaleva ${ }^{18}$, G.D. Patel ${ }^{53}$, M. Patel ${ }^{54}$, C. Patrignani ${ }^{20, j}$, A. Pearce ${ }^{55,50}$, A. Pellegrino ${ }^{42}$, G. Penso ${ }^{26, m}$, M. Pepe Altarelli ${ }^{39}$, S. Perazzini ${ }^{15, e}$, P. Perret ${ }^{5}$, L. Pescatore ${ }^{46}$, K. Petridis ${ }^{47}$, A. Petrolini ${ }^{20, j}$, M. Petruzzo ${ }^{22}$, E. Picatoste Olloqui ${ }^{37}$, B. Pietrzyk ${ }^{4}$, M. Pikies ${ }^{27}$, D. Pinci ${ }^{26}$, A. Pistone ${ }^{20}$, A. Piucci ${ }^{12}$, S. Playfer ${ }^{51}$, M. Plo Casasus ${ }^{38}$, T. Poikela ${ }^{39}$, F. Polci ${ }^{8}$, A. Poluektov ${ }^{49} 35$, I. Polyakov ${ }^{32}$, E. Polycarpo ${ }^{2}$, A. Popov ${ }^{36}$, D. Popov ${ }^{11,39}$, B. Popovici ${ }^{30}$, C. Potterat ${ }^{2}$, E. Price ${ }^{47}$, J.D. Price ${ }^{53}$, J. Prisciandaro ${ }^{38}$, A. Pritchard ${ }^{53}$, C. Prouve ${ }^{47}$, V. Pugatch ${ }^{45}$, A. Puig Navarro ${ }^{40}$, G. Punzi ${ }^{24, s}$, W. Qian ${ }^{56}$, R. Quagliani ${ }^{7,47}$, B. Rachwal ${ }^{27}$, J.H. Rademacker ${ }^{47}$, M. Rama ${ }^{24}$, M. Ramos Pernas ${ }^{38}$, M.S. Rangel ${ }^{2}$, I. Raniuk ${ }^{44}$, G. Raven ${ }^{43}$, F. Redi ${ }^{54}$, S. Reichert ${ }^{55}$, A.C. dos Reis ${ }^{1}$, V. Renaudin ${ }^{7}$, S. Ricciardi ${ }^{50}$, S. Richards ${ }^{47}$, M. Rihl ${ }^{39}$, K. Rinnert ${ }^{53,39}$, V. Rives Molina ${ }^{37}$, P. Robbe ${ }^{7,39}$, A.B. Rodrigues ${ }^{1}$, E. Rodrigues ${ }^{55}$, J.A. Rodriguez Lopez ${ }^{64}$, P. Rodriguez Perez ${ }^{55}$,

A. Rogozhnikov ${ }^{67}$, S. Roiser ${ }^{39}$, V. Romanovsky ${ }^{36}$, A. Romero Vidal ${ }^{38}$, J. W. Ronayne ${ }^{13}$ M. Rotondo ${ }^{23}$, T. Ruf ${ }^{39}$, P. Ruiz Valls ${ }^{68}$, J.J. Saborido Silva ${ }^{38}$, N. Sagidova ${ }^{31}$, B. Saitta ${ }^{16, f}$, V. Salustino Guimaraes ${ }^{2}$, C. Sanchez Mayordomo ${ }^{68}$, B. Sanmartin Sedes ${ }^{38}$, R. Santacesaria ${ }^{26}$, C. Santamarina Rios ${ }^{38}$, M. Santimaria ${ }^{19}$, E. Santovetti ${ }^{25, l}$, A. Sarti ${ }^{19, m}$, C. Satriano ${ }^{26, n}$, 
A. Satta ${ }^{25}$, D.M. Saunders ${ }^{47}$, D. Savrina ${ }^{32,33}$, S. Schael ${ }^{9}$, M. Schiller ${ }^{39}$, H. Schindler ${ }^{39}$, M. Schlupp ${ }^{10}$, M. Schmelling ${ }^{11}$, T. Schmelzer ${ }^{10}$, B. Schmidt ${ }^{39}$, O. Schneider ${ }^{40}$, A. Schopper ${ }^{39}$, M. Schubiger ${ }^{40}$, M.-H. Schune ${ }^{7}$, R. Schwemmer ${ }^{39}$, B. Sciascia ${ }^{19}$, A. Sciubba ${ }^{26, m}$, A. Semennikov ${ }^{32}$, A. Sergi ${ }^{46}$, N. Serra ${ }^{41}$, J. Serrano ${ }^{6}$, L. Sestini ${ }^{23}$, P. Seyfert ${ }^{21}$, M. Shapkin ${ }^{36}$, I. Shapoval ${ }^{17,44, g}$, Y. Shcheglov ${ }^{31}$, T. Shears ${ }^{53}$, L. Shekhtman ${ }^{35}$, V. Shevchenko ${ }^{66}$, A. Shires ${ }^{10}$, B.G. Siddi ${ }^{17}$, R. Silva Coutinho ${ }^{41}$, L. Silva de Oliveira ${ }^{2}$, G. Simi $^{23, s}$, M. Sirendi ${ }^{48}$, N. Skidmore ${ }^{47}$, T. Skwarnicki ${ }^{60}$, E. Smith ${ }^{54}$, I.T. Smith ${ }^{51}$, J. Smith ${ }^{48}$, M. Smith ${ }^{55}$, H. Snoek ${ }^{42}$, M.D. Sokoloff ${ }^{58,39}$, F.J.P. Soler ${ }^{52}$, F. Soomro ${ }^{40}$, D. Souza ${ }^{47}$, B. Souza De Paula ${ }^{2}$, B. Spaan ${ }^{10}$, P. Spradlin ${ }^{52}$,

S. Sridharan ${ }^{39}$, F. Stagni ${ }^{39}$, M. Stahl ${ }^{12}$, S. Stahl ${ }^{39}$, S. Stefkova ${ }^{54}$, O. Steinkamp ${ }^{41}$, O. Stenyakin ${ }^{36}$, S. Stevenson ${ }^{56}$, S. Stoica ${ }^{30}$, S. Stone ${ }^{60}$, B. Storaci ${ }^{41}$, S. Stracka ${ }^{24, t}$, M. Straticiuc ${ }^{30}$, U. Straumann ${ }^{41}$, L. Sun ${ }^{58}$, W. Sutcliffe ${ }^{54}$, K. Swientek ${ }^{28}$, S. Swientek ${ }^{10}$, V. Syropoulos ${ }^{43}$, M. Szczekowski ${ }^{29}$, T. Szumlak ${ }^{28}$, S. T'Jampens ${ }^{4}$, A. Tayduganov ${ }^{6}$, T. Tekampe ${ }^{10}$, G. Tellarini ${ }^{17, g}$,

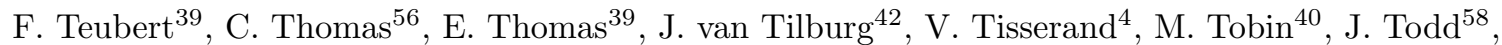
S. Tolk ${ }^{43}$, L. Tomassetti ${ }^{17, g}$, D. Tonelli ${ }^{39}$, S. Topp-Joergensen ${ }^{56}$, E. Tournefier ${ }^{4}$, S. Tourneur ${ }^{40}$,

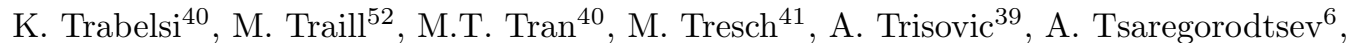
P. Tsopelas ${ }^{42}$, N. Tuning ${ }^{42,39}$, A. Ukleja ${ }^{29}$, A. Ustyuzhanin ${ }^{67,66}$, U. Uwer ${ }^{12}$, C. Vacca ${ }^{16,39, f}$, V. Vagnoni ${ }^{15}$, G. Valenti ${ }^{15}$, A. Vallier ${ }^{7}$, R. Vazquez Gomez ${ }^{19}$, P. Vazquez Regueiro ${ }^{38}$, C. Vázquez Sierra ${ }^{38}$, S. Vecchi ${ }^{17}$, M. van Veghel ${ }^{42}$, J.J. Velthuis ${ }^{47}$, M. Veltri ${ }^{18, h}$, G. Veneziano ${ }^{40}$, M. Vesterinen ${ }^{12}$, B. Viaud ${ }^{7}$, D. Vieira ${ }^{2}$, M. Vieites Diaz ${ }^{38}$, X. Vilasis-Cardona ${ }^{37, p}$, V. Volkov ${ }^{33}$, A. Vollhardt ${ }^{41}$, D. Voong ${ }^{47}$, A. Vorobyev ${ }^{31}$, V. Vorobyev ${ }^{35}$, C. Voß ${ }^{65}$, J.A. de Vries ${ }^{42}$, R. Waldi ${ }^{65}$, C. Wallace ${ }^{49}$, R. Wallace ${ }^{13}$, J. Walsh ${ }^{24}$, J. Wang ${ }^{60}$, D.R. Ward ${ }^{48}$, N.K. Watson ${ }^{46}$, D. Websdale ${ }^{54}$, A. Weiden ${ }^{41}$, M. Whitehead ${ }^{39}$, J. Wicht ${ }^{49}$, G. Wilkinson ${ }^{56,39}$, M. Wilkinson ${ }^{60}$, M. Williams ${ }^{39}$, M.P. Williams ${ }^{46}$, M. Williams ${ }^{57}$, T. Williams ${ }^{46}$, F.F. Wilson ${ }^{50}$, J. Wimberley ${ }^{59}$, J. Wishahi ${ }^{10}$, W. Wislicki ${ }^{29}$, M. Witek ${ }^{27}$, G. Wormser ${ }^{7}$, S.A. Wotton ${ }^{48}$, K. Wraight ${ }^{52}$, S. Wright ${ }^{48}$, K. Wyllie $^{39}$, Y. Xie ${ }^{63}$, Z. Xu ${ }^{40}$, Z. Yang ${ }^{3}$, H. Yin ${ }^{63}$, J. Yu ${ }^{63}$, X. Yuan ${ }^{35}$, O. Yushchenko ${ }^{36}$, M. Zangoli ${ }^{15}$, M. Zavertyaev ${ }^{11, c}$, L. Zhang ${ }^{3}$, Y. Zhang ${ }^{3}$, A. Zhelezov ${ }^{12}$, Y. Zheng ${ }^{62}$, A. Zhokhov ${ }^{32}$, L. Zhong ${ }^{3}$, V. Zhukov ${ }^{9}$, S. Zucchelli ${ }^{15}$

1 Centro Brasileiro de Pesquisas Físicas (CBPF), Rio de Janeiro, Brazil

2 Universidade Federal do Rio de Janeiro (UFRJ), Rio de Janeiro, Brazil

3 Center for High Energy Physics, Tsinghua University, Beijing, China

4 LAPP, Université Savoie Mont-Blanc, CNRS/IN2P3, Annecy-Le-Vieux, France

5 Clermont Université, Université Blaise Pascal, CNRS/IN2P3, LPC, Clermont-Ferrand, France

6 CPPM, Aix-Marseille Université, CNRS/IN2P3, Marseille, France

7 LAL, Université Paris-Sud, CNRS/IN2P3, Orsay, France

8 LPNHE, Université Pierre et Marie Curie, Université Paris Diderot, CNRS/IN2P3, Paris, France

9 I. Physikalisches Institut, RWTH Aachen University, Aachen, Germany

10 Fakultät Physik, Technische Universität Dortmund, Dortmund, Germany

11 Max-Planck-Institut für Kernphysik (MPIK), Heidelberg, Germany

12 Physikalisches Institut, Ruprecht-Karls-Universität Heidelberg, Heidelberg, Germany

13 School of Physics, University College Dublin, Dublin, Ireland

14 Sezione INFN di Bari, Bari, Italy

15 Sezione INFN di Bologna, Bologna, Italy

16 Sezione INFN di Cagliari, Cagliari, Italy

17 Sezione INFN di Ferrara, Ferrara, Italy

18 Sezione INFN di Firenze, Firenze, Italy

19 Laboratori Nazionali dell'INFN di Frascati, Frascati, Italy

20 Sezione INFN di Genova, Genova, Italy

21 Sezione INFN di Milano Bicocca, Milano, Italy 
24 Sezione INFN di Pisa, Pisa, Italy

25 Sezione INFN di Roma Tor Vergata, Roma, Italy

26 Sezione INFN di Roma La Sapienza, Roma, Italy

27 Henryk Niewodniczanski Institute of Nuclear Physics Polish Academy of Sciences, Kraków, Poland AGH - University of Science and Technology, Faculty of Physics and Applied Computer Science, Kraków, Poland

29 National Center for Nuclear Research (NCBJ), Warsaw, Poland

30 Horia Hulubei National Institute of Physics and Nuclear Engineering, Bucharest-Magurele, Romania

31 Petersburg Nuclear Physics Institute (PNPI), Gatchina, Russia

32 Institute of Theoretical and Experimental Physics (ITEP), Moscow, Russia

33 Institute of Nuclear Physics, Moscow State University (SINP MSU), Moscow, Russia

34 Institute for Nuclear Research of the Russian Academy of Sciences (INR RAN), Moscow, Russia

35 Budker Institute of Nuclear Physics (SB RAS) and Novosibirsk State University, Novosibirsk, Russia

36 Institute for High Energy Physics (IHEP), Protvino, Russia

37 Universitat de Barcelona, Barcelona, Spain

38 Universidad de Santiago de Compostela, Santiago de Compostela, Spain

39 European Organization for Nuclear Research (CERN), Geneva, Switzerland

40 Ecole Polytechnique Fédérale de Lausanne (EPFL), Lausanne, Switzerland

41 Physik-Institut, Universität Zürich, Zürich, Switzerland

42 Nikhef National Institute for Subatomic Physics, Amsterdam, The Netherlands

43 Nikhef National Institute for Subatomic Physics and VU University Amsterdam, Amsterdam, The Netherlands

44 NSC Kharkiv Institute of Physics and Technology (NSC KIPT), Kharkiv, Ukraine

45 Institute for Nuclear Research of the National Academy of Sciences (KINR), Kyiv, Ukraine

46 University of Birmingham, Birmingham, United Kingdom

47 H.H. Wills Physics Laboratory, University of Bristol, Bristol, United Kingdom

48 Cavendish Laboratory, University of Cambridge, Cambridge, United Kingdom

49 Department of Physics, University of Warwick, Coventry, United Kingdom

50 STFC Rutherford Appleton Laboratory, Didcot, United Kingdom

51 School of Physics and Astronomy, University of Edinburgh, Edinburgh, United Kingdom

52 School of Physics and Astronomy, University of Glasgow, Glasgow, United Kingdom

53 Oliver Lodge Laboratory, University of Liverpool, Liverpool, United Kingdom

54 Imperial College London, London, United Kingdom

55 School of Physics and Astronomy, University of Manchester, Manchester, United Kingdom

56 Department of Physics, University of Oxford, Oxford, United Kingdom

57 Massachusetts Institute of Technology, Cambridge, MA, United States

58 University of Cincinnati, Cincinnati, OH, United States

59 University of Maryland, College Park, MD, United States

60 Syracuse University, Syracuse, NY, United States

61 Pontifícia Universidade Católica do Rio de Janeiro (PUC-Rio), Rio de Janeiro, Brazil, associated $t^{2}$

62 University of Chinese Academy of Sciences, Beijing, China, associated to ${ }^{3}$

63 Institute of Particle Physics, Central China Normal University, Wuhan, Hubei, China, associated to $^{3}$

64 Departamento de Fisica, Universidad Nacional de Colombia, Bogota, Colombia, associated to ${ }^{8}$

65 Institut für Physik, Universität Rostock, Rostock, Germany, associated to ${ }^{12}$

66 National Research Centre Kurchatov Institute, Moscow, Russia, associated to ${ }^{32}$

67 Yandex School of Data Analysis, Moscow, Russia, associated to ${ }^{32}$ 
68 Instituto de Fisica Corpuscular (IFIC), Universitat de Valencia-CSIC, Valencia, Spain, associated $t^{37}$

69 Van Swinderen Institute, University of Groningen, Groningen, The Netherlands, associated to ${ }^{42}$

a Universidade Federal do Triângulo Mineiro (UFTM), Uberaba-MG, Brazil

${ }^{b}$ Laboratoire Leprince-Ringuet, Palaiseau, France

c P.N. Lebedev Physical Institute, Russian Academy of Science (LPI RAS), Moscow, Russia

d Università di Bari, Bari, Italy

e Università di Bologna, Bologna, Italy

$f$ Università di Cagliari, Cagliari, Italy

$g$ Università di Ferrara, Ferrara, Italy

$h$ Università di Urbino, Urbino, Italy

i Università di Modena e Reggio Emilia, Modena, Italy

j Università di Genova, Genova, Italy

${ }^{k}$ Università di Milano Bicocca, Milano, Italy

${ }^{l}$ Università di Roma Tor Vergata, Roma, Italy

$m$ Università di Roma La Sapienza, Roma, Italy

$n$ Università della Basilicata, Potenza, Italy

o AGH - University of Science and Technology, Faculty of Computer Science, Electronics and Telecommunications, Kraków, Poland

$p$ LIFAELS, La Salle, Universitat Ramon Llull, Barcelona, Spain

$q$ Hanoi University of Science, Hanoi, Viet Nam

$r$ Università di Padova, Padova, Italy

$s$ Università di Pisa, Pisa, Italy

${ }^{t}$ Scuola Normale Superiore, Pisa, Italy

${ }^{u}$ Università degli Studi di Milano, Milano, Italy ${ }^{\dagger}$ Deceased 